\title{
Towards a global participatory platform
}

\section{Democratising open data, complexity science and collective intelligence}

S. Buckingham Shum ${ }^{1}$, K. Aberer ${ }^{2}$, A. Schmidt ${ }^{3}$, S. Bishop ${ }^{4}$, P. Lukowicz ${ }^{5}$,

S. Anderson ${ }^{6}$, Y. Charalabidis ${ }^{7}$, J. Domingue ${ }^{1}$, S. de Freitas ${ }^{8}$, I. Dunwell ${ }^{8}$,

B. Edmonds ${ }^{9}$, F. Grey ${ }^{10}$, M. Haklay ${ }^{11}$, M. Jelasity ${ }^{12}$, A. Karpištšenko ${ }^{13}$,

J. Kohlhammer ${ }^{14}$, J. Lewis ${ }^{15}$, J. Pitt ${ }^{16}$, R. Sumner ${ }^{17}$, and D. Helbing ${ }^{18}$

${ }^{1}$ Knowledge Media Institute, The Open University, Milton Keyness, MK7 6AA, UK

2 Distributed Information Systems Laboratory, École Polytechnique Fédérale de Lausanne, EPFL-IC-IIF-LSIR, Bâtiment BC, Station 14, 1015 Lausanne, Switzerland

${ }^{3}$ Institut für Visualisierung und Interaktive Systeme, Universität Stuttgart, Universitätstraße 38, 70569 Stuttgart, Germany

${ }^{4}$ Dept. Mathematics, University College London, Gower Street, WC1E 6BT London, UK

${ }^{5}$ Embedded Systems Lab, University of Passau, IT-Zentrum/International House, Innstrasse 43, 94032 Passau, Germany

${ }^{6}$ School of Informatics, University of Edinburgh, Crichton Street, Edinburgh EH8 9AB, UK

${ }^{7}$ Information Systems Laboratory, University of the Aegean, Karlovasi, Samos 83200, Greece

${ }^{8}$ Serious Games Institute, Coventry Innovation Village, Coventry University Technology Park, Cheetah Road, Coventry CV1 2TL, UK

${ }^{9}$ Centre for Policy Modelling, Manchester Metropolitan University, Aytoun Building, Aytoun Street, Manchester M1 3GH, UK

10 Citizen Cyberscience Centre, CERN, UNOSAT, 211 Geneva, Switzerland

${ }^{11}$ Dept. Civil, Environmental and Geomatic Engineering, University College London, Gower Street WC1E 6BT, UK

12 Research Group on Artificial Intelligence, Hungarian Academy of Science and University of Szeged, PO Box 652, 6701 Szeged, Hungary

13 Skype Labs, Skype, Akadeemia tee 15b, Tallinn 12618, Estonia

${ }^{14}$ Fraunhofer-Institut für Graphische Datenverarbeitung IGD, Fraunhoferstr. 5, 64283 Darmstadt, Germany

15 Dept. Anthropology, University College London, 14 Taviton St, London WC1H, UK

${ }^{16}$ Dept. Electrical \& Electronic Engineering, Imperial College London, SW7 2BT London, UK

17 Disney Research Zurich, Clausiusstrasse 49, 8092 Zurich, Switzerland

18 ETH Zurich, Clausiusstraße 50, 8092 Zurich, Switzerland

Received in final form 9 October 2012

Published online 5 December 2012

Abstract. The FuturICT project seeks to use the power of big data, analytic models grounded in complexity science, and the collective intelligence they yield for societal benefit. Accordingly, this paper argues that these new tools should not remain the preserve of restricted 
government, scientific or corporate élites, but be opened up for societal engagement and critique. To democratise such assets as a public good, requires a sustainable ecosystem enabling different kinds of stakeholder in society, including but not limited to, citizens and advocacy groups, school and university students, policy analysts, scientists, software developers, journalists and politicians. Our working name for envisioning a sociotechnical infrastructure capable of engaging such a wide constituency is the Global Participatory Platform (GPP). We consider what it means to develop a GPP at the different levels of data, models and deliberation, motivating a framework for different stakeholders to find their ecological niches at different levels within the system, serving the functions of (i) sensing the environment in order to pool data, (ii) mining the resulting data for patterns in order to model the past/present/future, and (iii) sharing and contesting possible interpretations of what those models might mean, and in a policy context, possible decisions. A research objective is also to apply the concepts and tools of complexity science and social science to the project's own work. We therefore conceive the global participatory platform as a resilient, epistemic ecosystem, whose design will make it capable of selforganization and adaptation to a dynamic environment, and whose structure and contributions are themselves networks of stakeholders, challenges, issues, ideas and arguments whose structure and dynamics can be modelled and analysed.

\section{Vision}

The highest aim of FuturICT is to build better ways to address the urgent, systemic problems now threatening the sustainability of societies at many scales. The priority of the particular project strand that this paper focuses on is the development of "Collective Intelligence" (CI), which the inaugural conference devoted to computersupported CI defines as:

"... behaviour that is both collective and intelligent. By collective, we mean groups of individual actors, including, for example, people, computational agents, and organizations. By intelligent, we mean that the collective behaviour of the group exhibits characteristics such as, for example, perception, learning, judgment, or problem solving." 1

In the Harvard 2010 Symposium on Hard Problems in Social Science, of the problems proposed by the panel, three of the top six voted "extremely important" connect directly with this: Increasing collective wisdom, Aggregating information and Knowledge acquisition.

In the historical context of computer-supported intellectual work, FuturICT traces its roots back to Douglas Engelbart's [29] ground-breaking programme to invent new computational tools to "augment human intellect" and "Collective IQ" in order to tackle society's "complex urgent problems." Engelbart's innovations included the mouse, hypertext, real-time electronic text/graphics editing, and established the foundational concepts for the personal computing paradigm, in which computers became interactive enough, at both the physical and cognitive ergonomic levels, to serve as personal tools for thought: to manipulate "concept structures" (i.e. symbolic representations of worlds in text and graphics), to annotate sources, connect ideas, deliberate and debate, and ultimately, to make better decisions.

The largely unfulfilled dimension of Engelbart's vision was what might be possible collaboratively when the tools became an everyday commodity, and a critical mass of

\footnotetext{
1 wWw.ci2012.org
} 
people became literate with these new tools for reading and writing. A half-century later, with those same, persistent societal problems as our focus, FuturICT's mission is to help shape the collective computing paradigm, equipping different scales of collective agent to more effectively sense their environments, interpret signals, manipulate symbolic representations of the world, annotate, connect, deliberate and debate, and ultimately, make better decisions.

\subsection{Goals}

The paper in this special issue by van den Hoven, et al. [77] sets out the ethical imperative for a project such as FuturICT, identifying four different arguments for moving societal data and analytical tools that may shape decision making, into an open, participatory paradigm:

(1) Epistemic Responsibility: Those who bear responsibility for policies and interventions in complex systems have a (higher order) responsibility for creating the knowledge conditions which allow them to do the best they can. Decision makers are framed by a given epistemic context and are dependent on the information infrastructure put at their disposal. The quality of their decisions and judgments is in many cases determined by the quality of their knowledge tools (i.e., information systems, programs and data). Responsibility of decision makers therefore importantly concerns the design ex ante of epistemic resources and information infrastructures, which is a major aim of FuturICT.

(2) Social Knowledge as a Public Good: A broad range of information about society ought to be accessible to all citizens under conditions of equal opportunity. FuturICT forms a counter-balance against the buildup of information monopolies in important domains in society by private sector companies and thus contributes to a just and fair information society.

(3) Privacy by Design: Privacy is an essential moral constraint for achieving knowledge and understanding of social reality in information societies. Although the term refers to a broad range of moral rights, needs, claims, interests, and responsibilities concerning (information about) the person, personal lives, and personal identity, privacy is essential for the flourishing of individual human beings. Data protection technology needs to be developed in tandem with data mining techniques and Esocial science. The development of new forms of Privacy by Design is a central objective of FuturICT.

(4) Trust in Information Society: Trust implies a moral relationship between the truster and the trustee, a relationship that is partly constituted by a belief or an assumption that the trustee will act from the moral point of view. In complex ICT-shaped environments trust requires that those in charge of the design of the environment, in which the trust relationship is situated, are as explicit and transparent as possible about the values, principles and policies that have guided them in design. This is a fourth guiding principle for FuturICT, whose ultimate goal is the fair information society, where there is openness and transparency about the values, principles and policies that shape it.

The purpose of this paper is to consider what it means to take seriously such arguments. In other words, how to facilitate the development of knowledge, both opening up and easing interaction between contributors to this process? The answer that this paper proposes is to develop a "Global Participatory Platform" (GPP). This would be a socio-technical infrastructure that enabled the open collaboration and combination of all the elements that go into directing and making useful knowledge. This would include: provision of data sets, analysis, data-mining, complex modeling and simulation, visualisation, deliberation, discussion, collective decision-making and feedback. 


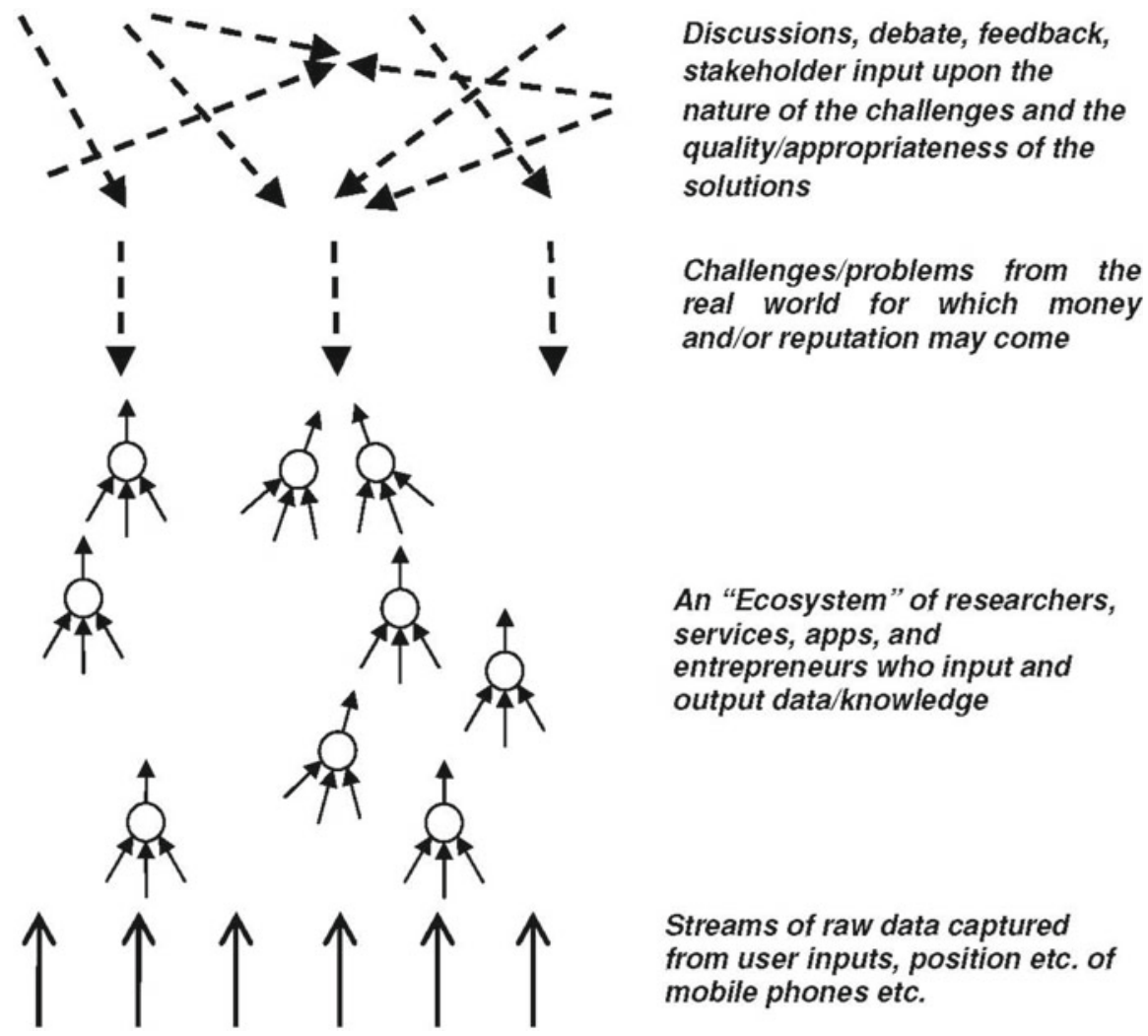

Fig. 1. Conceiving the Global Participatory Platform as an Information Ecosystem.

In this way the GPP would open up and democratise the development and use of knowledge, releasing the potential synergies between these elements and hence better deliver the public good of sound knowledge and good decision making to equal the challenges of social complexity and uncertainty that the world faces.

The GPP would be a coherent set of interfaces, services, software infrastructures, tools, and APIs as well as social institutions, legal and social norms that would allow the participants to collaborate openly, freely and creatively in the development and use of knowledge. It would comprise an open platform on which it will be easy to build both non-commercial and commercial applications, services, projects and organizations. Its inputs would be the data, models, tools, simulations, hypotheses, needs, questions and opinions that the various stakeholders would develop and supply. Its outputs would be analyses, knowledge, collaborative projects, and collective decisions as well as new: questions, needs, issues and directions. In summary the whole system could be thought of as a flexible and dynamic informational ecosystem whereby all participants can find their ecological niche by both meeting their own needs and, as a consequence, contributing to the whole system and hence the wider public good (Fig. 1). This concept is discussed further in Sect. 3.3.

The kinds of properties that the ecosystem created by the GPP should display, and which are explored in this paper, include:

- transparency of data sources, algorithms, and platform use

- control of users over their personal data

- privacy-respecting data mining

- self-regulation, self-healing 
- reliability and resilience

- promotion of constructive social norms and responsible use

- crowd-based monitoring of platform use, involving non-profit organizations

- tools to alert problems and conflicts, and to help solving them

- incentives to share profits generated from data and algorithms provided by users

- mechanisms for managing unethical use.

The plausibility of this proposal rests on its feasibility. How exactly does one design such a system? How might one reconcile the needs of privacy and open access? How would the discursive and analysis aspects of the system combine? How does one best facilitate synergy between participants? How does one make the system as accessible as possible, yet retain scientific credibility? It is these kinds of questions that this paper addresses.

\subsection{Opportunities}

FuturICT differs in an important respect from other well known 'big science' projects. Neither the Large Hadron Collider nor the Human Genome project expected active engagement from non-experts, and understandably so: they probably would not have benefited from it scientifically, given the esoteric nature of the science. However, in contrast to the Higgs boson or DNA sequences, the 'objects of enquiry' in FuturICT are sentient beings who are concerned about how they are studied, what decisions might be made based on data about them, and whether those decisions are justified. Moreover, since citizens might themselves access this data, reflect on their situation and environment, and consequently modify their behaviour, we are dealing with feedback loops in which the observed observe their observers, with all agents continuously adapting. FuturICT's distinctive combination of the complexity, social and computing sciences seeks to devise appropriate ways to design and evolve socially aware infrastructure that recognizes such complexity.

An important debate must therefore be opened up around access to these tools, which, we propose, are potentially as revolutionary in how we read and write meaning as the shift from orality to literacy [47] and the democratisation of printed books [28]. Learning from the lessons of the Gutenberg revolution and the spread of literacy, to many people it seems antiquated, and even morally untenable, to argue that literacy with the new tools, and access to the new digital libraries, should remain the preserve of an élite for fear that 'the uneducated masses' cannot be entrusted with such power. On the other hand, others will argue that digital datasets and social simulations are qualitatively different from their paper predecessors, such that only a responsible élite can be trusted to use them responsibly: naively opening up such tools to public access brings huge risks of abuse from businesses and criminals. Challenging those who would maintain the walled gardens, will be those who see predominantly open systems and data as the only way forward.

In this unfolding landscape, citizens at large may wonder if it is scaremongering to worry about the risk of a 'Big Brother' scenario, in which the models and forecasts made possible by such an infrastructure remain the preserve of a scientific and political élite, further undermining trust in such institutions. Moreover, might this not lead to gaming of the system by citizens?

While FuturICT can and will consider these issues theoretically, the initiative is distinctive in also having the capacity to prototype and study future infrastructures, in order to answer these questions empirically. Is it possible to make these new tools accessible, comprehensible, debatable and shaped by as many as possible? Moving beyond armchair thought experiments, what reactions and behaviours do they elicit 
when actually placed in the hands of citizens, scientists or policymakers? The revolutionary impact of mobiles, and now smartphones, demonstrates that many people are happy to reap the benefits of heavily marketed products with little concern about their personal data, happy to leave it to others to grapple with the complexities of the law and ethics. Perhaps the most immediate risk is that most citizens have not grasped the shift that is underway, or are so disengaged or disempowered, that they simply do not care what happens to their personal data, or that decisions could be made about their lives based on flawed models grounded in untrustworthy data. The ambition of democratising big data, modelling and the insights they yield, brings with it some very complex challenges. To make such assets a public good, requires a sustainable ecosystem enabling different kinds of stakeholder in society to engage, including but not limited to, citizens and advocacy groups, school and university students, policy analysts, scientists, software developers, journalists, politicians. Meaningful engagement covers intellectual and social processes such as understanding what the project is doing at a general level, grasping specific concepts (e.g. "emergence"; "positive feedback"), comprehending and interacting with visualisations, participating in and learning from serious games, sharing interpretations of documents, debating policy implications and contributing data, models and tools.

The possible futures we can envisage may challenge our notions of privacy, redefine the meaning of informed consent in the context of open data, and redraw the boundaries between what is legal and what is ethical. There will be new literacies associated with reading and writing meaning in these new tools, which instill better understanding of the responsible use of datasets, simulations and visualisations, which can obfuscate as well as illuminate.

\subsection{User scenarios}

We will give a number of examples throughout this paper, but we open with three user scenarios designed to illustrate some of the key ideas to be elaborated: citizen benefits and engagement from children upwards; information visualization services; collectively contributed, curated and shared data; participatory deliberation and multiplayer gaming at scale; science education; policy advice; free and commercial services built over this infrastructure.

\subsubsection{The primary school's H1N1 observatory}

Alessandro Vespignani (one of FuturICT's partners) was able to model accurately the spread of H1N1 through mathematical models of infection combined with global travel data (http://www.gleamviz.org). Inspired by this, Ms. Teacher in Little Village challenges her 11 year old students to set up an observatory to predict how soon H1N1 would reach Little Village, given outbreaks in the nearest city 10 miles away, and several locations around the world, and to demonstrate their understanding of why they reach the conclusions they do. The students build their H1N1 portal using the GPP web toolkit to drag and drop a set of widgets together to interrogate static and live datasets, mash them up using rules defined in a simple visual language, and then render the results using a range of visualisation widgets. They also devise a sensor network game in which villagers "infect" each other via their phones when they meet under certain conditions, allowing them to study the spread of the disease within their own school and local streets, which really drives home the seriousness of the illness. The conclusions are not definitive, so they summarise policy recommendations to their Minister for Health using argument maps to distill on a single page the key 
issues for deliberation, the tradeoffs between different options, and the evidence-base underpinning each one. Hyperlinks in the maps reveal more detail on request, showing different states in the simulation models and visualisations at which key turning points are judged to be seen, with automatically generated textual narratives summarising the key assumptions, variables and dependencies.

\subsubsection{The Cats+Tremors Observatory}

Cat lovers build the Cats+Tremors social network in a GPP-powered online space, convinced that it's not only dogs who can detect earthquakes before human sensors. They self-organise to monitor their beloved pets' behaviour, sharing videos and event diaries, using a common coding scheme they have evolved themselves, embedded in a phone app they collectively fund-raised to have built. This uploads data in a common format to the GPP, which enables very large scale data fusion, authenticated timestamping (to prevent retrospective fabrication of cat data), and validated statistical correlations after testing against verified geo-physical data from professional scientific institutions, visualised in a variety of formats, with SMS alerts going out when the model's thresholds are exceeded. A public website shares the predictions in an open manner exposing the hypothesis to public scrutiny. Cat movies can be analysed using an open source, collaborative video-annotation tool. The assumptions built into the experiment are the subject of ongoing debate in the network, and several university teams are now working with the network to use their passion as the basis for promoting deeper learning about statistics, probability, animal behaviour, qualitative data analysis, and scientific reasoning.

\subsubsection{The Fitness Universe game}

The Fitness Universe Game utilises the GPP to bring together a wide range of stakeholders in a research-driven approach to adaptive problem solving. The connectivity of the GPP is leveraged to allow game developers to implement a wide range of different assets sourced semantically from the web within a game. In turn, these components allow for ethical data capture from players, and its subsequent analysis. This data is then used to refine the game, and inform policymakers of its impact. Where this differs from other adaptive gaming platforms is the power leveraged by the big data and complexity modelling techniques at the heart of FuturICT: adaptation is dynamic, flexible, and informed fully by an understanding of the data generated by not only the user base of the game, but also its contextual backdrop and links to other chains of cause and effect.

What kind of platform would need to be in place to deliver such scenarios? We use the concept of a "platform" to refer not only to digital technology, but more holistically, to include the motivations and skillsets that different stakeholders in society bring, and the practices they evolve as they appropriate technologies into their daily lives, as a means to many different ends. As we will see, when the ambition is to develop a participatory platform, the societal engagement issues are even more acute.

\subsection{The GPP in relation to FuturICT}

First, let us clarify in functional, technical terms how the Global Participatory Platform (GPP) is envisaged in relation to the other key elements of the FuturICT infrastructure, the Planetary Nervous System (PNS) and the Living Earth Simulator (LES) (Fig. 2). 


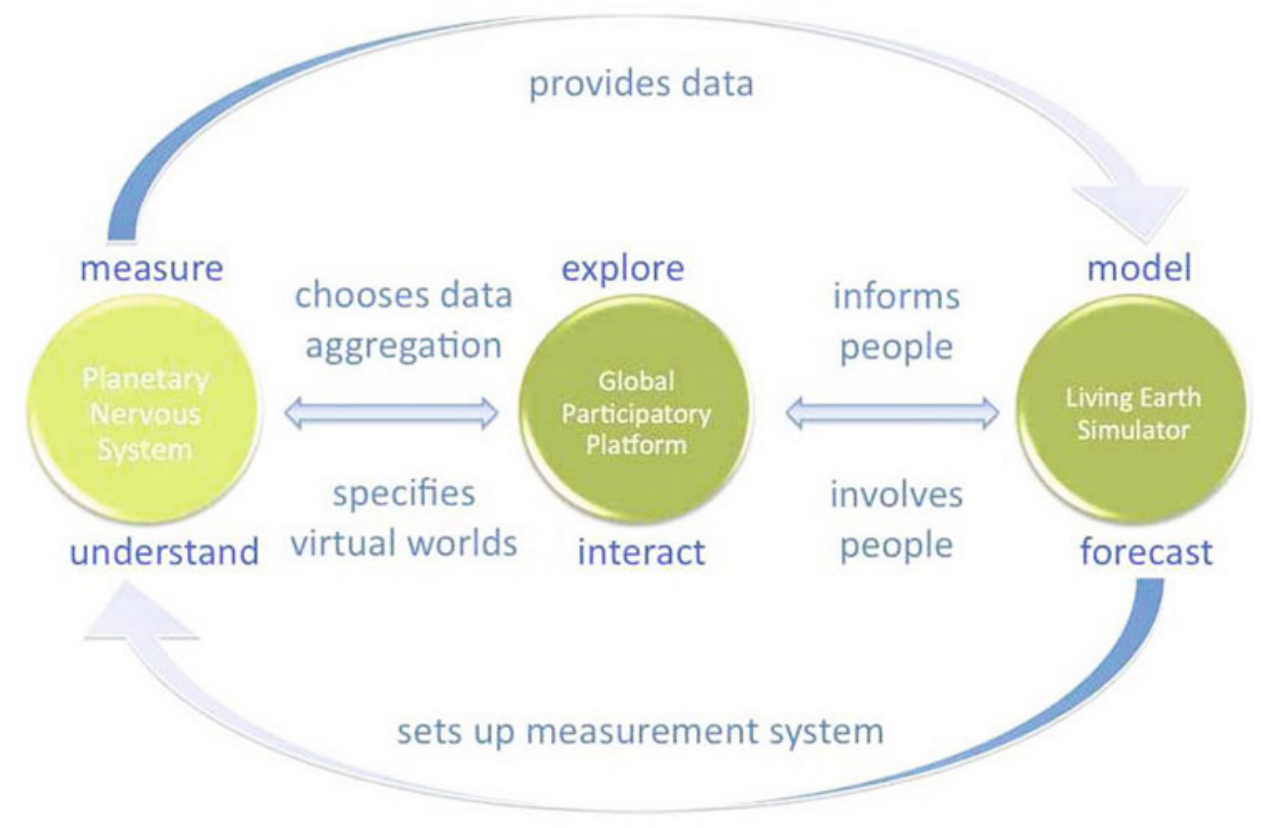

Fig. 2. The Global Participatory Platform as the interface between the Planetary Nervous System (PNS) and the Living Earth Simulator (LES).

The GPP is the interface between the Planetary Nervous System (sensor network) and the Living Earth Simulator (complex systems modelling), detailed in other papers in this special issue. Given a user query the PNS extracts relevant state information from all suitable data in the digital domain using mostly techniques from pattern analysis, data mining, knowledge discovery, and artificial intelligence in general. The information is then transformed into knowledge and predictions about possible futures by the LES using appropriate social science models and simulations. The process is highly interactive including continuous information flow between the PNS and the LES, iterative re-evaluation of models and data and involving the user through data presentation and exploration interfaces. Facilitating the above interaction between the user, the PNS and the LES is a key functionality of the GPP. The GPP is participatory in two key respects:

1. Making available to third party developers the methodologies, models, algorithms, libraries, etc. that will be developed to facilitate the work of the project's thematic Exploratories. We need to provide high level toolkits that empower a far wider user base, (see the primary school H1N1 observatory scenario). The GPP would ensure that proprietary data collected by the Exploratories would not be shared unethically.

2. Facilitating and brokering contributions from stakeholders including the public, scientists, computing centres, government agencies. Such contributions can be data, models, software, time, participation in serious games (or the right to observe gaming behaviour), and viewpoints in debates about policy implications. Thus a key component of the GPP will be a trustworthy, transparent, privacy respecting brokerage platform.

We distinguish three different types of digital data, each posing different challenges and each requiring different handling with respect to access rights, privacy and including it in the brokerage platform: 
1. Static data from organisational databases (e.g. governments, companies, NGOs, universities). This is the "traditional" source of data, released by professional entities with relatively clear usage constraints.

2. Dynamic data contributed by volunteers recruited for a specific cause. Examples would include mobile phone sensor traces, household automation (e.g. energy consumption) traces, personal records, social web entries, and responses to electronic questionnaires. This is the "participatory sensing" approach used by early Reality Mining work of for example Pentland [27].

3. Data "scavenged" from the openly available web. This includes public social media data (e.g. Twitter, Flickr, YouTube), digital news media, sensor information that is public (e.g. some people make their location data public, some traffic information and webcams are open), and public data about search query distribution and internet traffic. The huge quantities of real time data may make this an extraordinarily rich source of information, although the high noise-to-signal ratio remains an open research challenge. Data "scraped" from website texts designed primarily for human reading adds to the above.

Serious games can be seen as virtual worlds also providing data in the above categories: (1) data banks archiving past gaming behaviour, (2) volunteers playing specific games as their contribution to data collection, and (3) the mining of publicly available game traces. For details of the thinking in the Visioneer project preceding FuturICT, which has helped to shape the current paper, see Helbing, et al. [32].

\section{State of the art and open challenges}

While the democratisation of large datasets, simulation models and collective intelligence are potentially huge opportunities to carve new markets for small, medium and large businesses, and public institutions, this clearly carries the potential of undesirable and malicious use. Key risks include:

- Privacy violation, e.g., using private intelligence for theft

- Intellectual property violation, e.g. using private information for marketing purposes

- Misinformation, e.g. for inducing unfavourable buying decisions.

\subsection{Designing for trusted open data and services}

The idea of democratising different resources, mostly data, and democratising different processes, like gathering knowledge or solving problems is not new. The agenda that data generated by public organizations should be public has been promoted for almost as long, along with the idea that such data should be a basis of an ecosystem of applications that could use these datasets for the benefit of the public ${ }^{2}$.

What is new is scale, scope and complexity. Huge datasets introduce new challenges for democratisation, which we hypothesise will impact how we design future data models. One could argue that a centralised model of personal data is intrinsically undemocratic because access can be stopped at any time. What does it mean to "democratise" petabytes of data? Moreover, this challenge when confronted by a single data centre is entirely different to working with a fully distributed system storing the same data, or a hybrid system comprising a wide range of computing resources and database sizes. Requirements such as anonymisation, trust and resource sharing, and

\footnotetext{
${ }^{2}$ http://opendatachallenge.org
} 
abuses such as free-riding, all have different weights and imply different solutions in different data models. These need to be mapped to attributes of data models (e.g. level of distribution, archival integrity, availability, heterogeneity, ownership, encryption, load balancing).

In the sections that follow, we consider some of the key technological developments that enable the envisaged GPP commons (for data, models and interpretation), and how mechanisms might be designed into the GPP at many levels to address the abuses that may occur, in order to maintain the motivation for participation, and protect intellectual property, and privacy. We begin with community-level phenomena and requirements, and move gradually to examples of the technologies that may be capable of delivering these values.

\subsubsection{Community sensing}

The number of privately owned sensors is growing at a tremendous pace. Smartphones today harness not only GPS, but also sound-level, light and accelerometer sensors. Private weather stations are becoming connected to the Internet and in the near future we will also see increasing use of chemical sensors, e.g., for air quality monitoring.

Aggregating data from these diverse and plentiful sensor sources enables new forms of monitoring environmental and societal phenomena at an unprecedented scale and for a large variety of specialised applications that are of interest to communities of very different scales $[14,40]$. Some examples of such applications are monitoring the environmental footprint of citizens, assessing the health impact of environmental factors, traffic or crowd monitoring, physical reality games or the study of cultural and social phenomena.

Citizens owning these sensors are often willing to share the data provided that privacy concerns are properly addressed and that the social benefit is clearly identified. However, protecting privacy is far from trivial, as with powerful analysis and reasoning techniques impressive inferences can be made on the aggregate data [68]. Also sharing of data incurs for the citizens different costs, such as energy consumption on batteries, communication fees and sensor wear. Deploying and coordinating sensing campaigns considering these diverse requirements and aggregating and interpreting the resulting data are thus formidable engineering problems [1]. Key research challenges in community sensing concern:

- privacy protection in presence of inference and context information

- fair resource sharing models and incentive models to foster participation,

- distributed optimization and coordination of community sensing tasks

- aggregation of heterogeneous data from mobile sensors and model-based data processing.

A number of projects and research centers are addressing these questions from diverse perspectives such as the OpenSense (opensense.epfl.ch) or Hazewatch (pollution.ee.unsw.edu.au) projects on air quality monitoring in urban environments, the Urban Sensing lab (urban.cens.ucla.edu), the senseable city lab (senseable.mit.edu) and the MetroSense project (metrosense.cs.dartmouth.edu) investigating the use of mobile phones for various citizen oriented sensing tasks.

\subsubsection{Social contracts}

Given the above trends, we envisage that data in the GPP commons will be generated increasingly by individuals, currently explicitly: users volunteer their data, 
although when they sign up to some social networking sites, they are not always clear that they may be losing copyright, signing over their intellectual property, or what their privacy rights are. However, the technological developments of sensor networks, stream computing and communication channels mean that new content will be generated (for example, emotions, scent, brain-waves) through many new affordances, for example clothing, implants, prosthetics, and so on. The generation of this data is largely implicit, and an ethical issue of growing importance will concern the ease or difficulty with which citizens may opt-out of leaving a digital trail [30]. Therefore, we need to be more precise about a number of procedural and legal concepts related to the generation of implicit content, the social contract between generators and user of implicit content, and design guidelines for complexity modeling tools using implicit content. The procedural and legal concepts that need to be clarified include:

1. Ownership is a relationship between participants and content that implies that, legally, the owning participant decides about the possible use of the owned content.

2. Terms of use are the specification of which uses of owned content can by made in terms of limiting access to specific participants, specific times and specific conditions. This includes access control, the restriction of access to specific participants, preservation and deletion, and the restriction of access over time.

3. Control is the technical mechanism for enforcing the terms of use. This may include mechanisms to make unintended use technically unfeasible (e.g. using digital rights management), but also mechanisms to audit the use and thus produce proof of unintended use, which might be used in further legal procedures.

4. Agreements are made among different parties concerning the access to and use of information. They are usually legally binding.

5. Sanctions are technical or legal mechanisms applying in the event of the above being violated.

The social contract must be develop from a user-centric perspective, i.e. from the point of view of the content creators. Leveraging a user-centric position on digital rights management (DRM) it should be maintained that digital content should be 'sold' with whatever rules the creator/producer deems fit. For example, there is plenty of evidence that users will 'donate' their data to a charity for medical research, and in many other cases will exchange data and even rights in return for a service, especially if that service fills a pressing social need (e.g. Facebook). Whatever rules are specified, though, should be enforceable, provided:

- The rules themselves are not regressive. The Internet was founded on principles of maximising openness of connectivity and data transfer. It should not be exclusive to connect to the GPP and data transfer should not be supervised or regulated.

- Innovation in social networking is not stifled. Many artistic innovations spread from the bottom-up by word-of-mouth. Although it is delusional to suppose that social networking is an unstoppable force inevitably advancing democratic ideals and civil liberties [43], it remains a powerful opportunity to address global challenges like climate change.

- Technological invention is not prohibited. The Internet has been the source of many ideas being used for application for which they were not originally intended. Sometimes this has been for the general good (e.g. http which was the basis of the WWW), and sometimes not (smtp being used for spam), but whichever, the freedom to innovate should be protected.

- Narrowing of 'fair use' is not overly restrictive. There should be no prevention of copying for multiple players, archives, etc., nor should copying clipart for use in a school presentation be prevented. 
- There is no monopoly of tool producers. If there were only one 'trusted computing platform' and so content was only produced for that one platform, it would effectively extend a monopoly over software into a monopoly over content.

Therefore, content is associated with intellectual property rights, and these rights need to be managed on behalf of the content creators and producers, and respected by the content consumers. For example, downloading, and file sharing, are user actions that are not so much about the exchange of digital data, but the exchange of rights to use that data in certain ways, as expressed by a license or a contract. However, given the provisions expressed above, there should not be any centralised authority overseeing the enforcement of these rights: this means that conventional security mechanisms and top heavy (supply side) DRM techniques no longer apply. Instead, we need a new set of design guidelines.

Following Reynolds and Picard [58], who studied the issue of privacy in affective computing, we propose to ground those decisions on mutual agreement. The form of this agreement is a contract. Contractualism is the term used to describe philosophical theory that grounds morality, duty, or justice on a contract, often referred to as a social contract [56]. Reynolds and Picard extend this notion to Design Contractualism, whereby a designer makes a number of moral or ethical judgments and encodes them, more or less explicitly, in the system or technology. The more explicit the contract, the easier it is for the user to make an assessment of the designer's intentions and ethical decisions. There are already a number of examples of (implicit and explicit) design contractualism in software systems engineering, e.g. copyleft, ACM code of conduct, TRUSTe, and these need to replicated in the regulatory aspects of complexity modeling tools for the GPP.

\subsubsection{Avoiding a tragedy of the commons}

One approach to ensuring the stability of data in the GPP is to consider the GPP as a common pool resource, and take an institutional approach to its management. The motivation for this approach comes from Ostrom [49], who studied a variety of common pool resources in water, forestry and fishing, and found that in contrast to the "Tragedy of the Commons" predicted by a simple game-theoretic analysis, communities had managed to self-organise rule- and role-based systems which successfully managed and sustained the resource. Moreover, these systems institutions persisted as successive generations agreed to be bound by the same conventional rules, even though they had not been present at their original formulation. However, Ostrom also observed that there were some cases when these institutions endured, and some when they did not. She then identified eight principles as essential and determinate conditions for enduring institutions: (1) clearly defined boundaries to the resource and of institutional membership; (2) congruence of provision and appropriation rules to the state of the local environment; (3) collective choice arrangements are decided by those who are affected by them; (4) monitoring and enforcement of the rules is performed by the appropriators or agencies appointed by them; (5) graduated sanctions (i.e., more refined than 'one strike and you're out'); (6) access to fast, cheap conflict resolution mechanisms, and (7) the right to self-organise is not subject to interference from external authorities in how the community chooses to organise itself. (8) The final principle was systems of systems: that these self-organising institutions for self-governing the commons were part of a larger structure of nested enterprises.

Hess and Ostrom [33] proposed to analyse digital information in the Internet era from the perspective of a knowledge commons. Using the eight principles identified above, a design and analytical framework was proposed for understanding and treating knowledge as shared resource with social and ecological dimension. It could be 
argued that Wikipedia is an unplanned but fine example of these principles in action: but what is required for the GPP is a planned and principled operationalisation of these principles. In particular, one can see that incentives to contribute, and reciprocity of contribution, are encapsulated by the principles for congruence of provision and appropriation rules and self-detemination of the collective-choice rules. Notions of fairness, however this is measured, can be encapsulated by the sanctioning and conflict resolution rules. Furthermore, the clearly-defined boundaries and monitoring principles offer some protection against 'poisoning the data well', for example by the 'merchants of doubt' identified by Oreskes and Conway [48].

\subsubsection{Incentivising institutional data sharing}

An important part of the GPP ecosystem to understand is what incentivises institutions to share their data. The core business of the largest ICT companies is based almost exclusively on the private ownership of huge databases (e.g. of user behaviour and preferences, used to target and personalise services), so there is no incentive to share. Even in cases where data might be appropriately shared without violating privacy, there are technical difficulties in sharing the contents of data centers of several petabytes. Replicating is not an option, and accessing these data centers by the public is not an option due to cost.

Could they be incentivised to share commercially owned data, following the analogy of open source software (OSS)? In OSS, many private companies contribute significant resources to create products that in turn become available to the public (for example, the Suse and Redhat distributions of the open source Linux operating system, or Google's distribution of the Android operating system). The incentives for that certainly involve seeing software as a part of an infrastructure on top of which they can deliver paid services. In this case the company is interested in the diffusion of the software as widely as possible, so that their associated services can be sold in larger volumes, or to create cheap competition against rival, for-fee products. If the core business of a company is built on selling or owning the software itself, then there is very little incentive for them to contribute in the way they do. Given that commercial investment in OSS has proven to be a sustainable proposition, the question is whether commercial data sharing can draw inspiration from this in any way. Open data, however, is different from OSS. It is harder to see how sharing data under an open license could have the same commercial return, although by analogy, perhaps new forms of market can be developed which depend on consumers having ready access to the company's open data. In an information market where attention is the scarce resource, if open data draws more potential clients' eyes and maintains brand awareness, it has a value, both monetary and less tangible.

Corporate social responsibility could incentivise (at least some) corporations to increase data sharing, especially if there is a cultural shift in expectations around openness, and we witness a similar paradigm shift to what we are now seeing in scientific communication and datasets (e.g. to accelerate medical innovation, or environmental survival). Public institutions play an intermediary role in this engagement. While on the one hand, they have a vested interest in preserving and even increasing their institutional power, and as a result could exhibit a similar incentive structure to large corporations, on the other hand, their role is to serve as the aggregator of interests from different parts of society, including minority voices and the general interests of citizens. On occasions, of course, public institutions are called to defend these from commercial interests.

Looking ten years ahead, corporate incentives may change drastically if clients' interests also shift in unpredictable new ways. At present, the value proposition to 
consumers is defined by factors such as providing personally relevant and high quality information, preserving ownership and control over personal data, protecting privacy and against data fraud. We must remain open to the possibility that these values might be better satisfied in new ways that make use of open data.

\subsubsection{Prevention and sanctioning technologies}

Historically, two main technological approaches have been developed for tackling the abuses that the GPP might make possible: prevention and sanctioning.

Prevention seeks to avoid potential abuses a priori. Preventive measures to protect against misbehavior and the fraud on the Internet have been broadly studied. We can identify the following approaches that have been taken.

Cryptographic techniques: this approach aims at increasing the technical difficulty or cost in obtaining unauthorised access to data. For content and data sharing the most obvious use of such techniques is to distributed sensitive information only in encrypted forms. Drawbacks of cryptographic techniques are that they typically require, often complex, mechanisms for key sharing.

Obfuscation techniques: this approach aims at reducing the information content such that sensitive information is not published at all and that even with aggregation and inference attackers cannot derive sensitive information. Drawbacks of obfuscation techniques are that the value of the published information might be significantly diminished.

Reputation techniques: this approach aims at evaluating the earlier behavior of other agents in the system, for example information recipients, for assessing their trustworthiness using statistical and machine learning methods. Drawbacks of reputation techniques are that they may produce erroneous assessments and therefore unintended information disclosures may occur.

It is worth noting that the realization of these techniques, in particular the latter two, often rely on data analytics methods. Nevertheless, whatever technical means are chosen to prevent abuse, total security remains an elusive goal. Moreover, viewpoints on what constitutes acceptable behavior, and what is considered as abuse, depend on the societal context.

Sanctioning is a complementary mechanism for a community to promote acceptable behaviors. Sanctioning mechanisms do not a priori prevent misbehavior, but introduce sanctions a posteriori. The underlying hypothesis is that assuming 'rational behaviour' that does not enjoy sanctions this will serve as a deterrent. Sanctions should be community designed, making them a more 'democratic' control mechanism than technically enforced prevention, which can be harder to modify (although we can envisage end-user customisable prevention mechanisms for online spaces).

Sanctioning mechanisms will rely on data analytics in order to trace and analyse community activity we can see therefore how 'low level' design decisions about system logging will have escalating effects up to much higher level constructs such as 'managing appropriate behaviour'. Both preventive and sanctioning mechanisms rely on data analysis on earlier actions, which introduces the problem of identity verification.

\subsubsection{Identity and reputation}

Reliable identification is a core enabling mechanism for establishing trust [78]. Identity is the basic mechanism to link different pieces of information together. Identities are required both for content and participants. Reliable identification of participants is 
at the heart of every mechanism underlying a Trusted Web, but identity only assists with the problem of trust, if one can be sure that agents with poor reputation, or threatened with sanctions, cannot simply reinvent their identity ('whitewashing').

Signalling approaches to building reputation profiles [22] are based on analysis of past behaviour, models and measures that are inferred from past data, and prediction models that extrapolate such behavior into the future. In this way participants can decide whether or not to enter into an interaction in other words, whether they trust it. This approach underlies many works on robust recommendation and reputation systems. Whether applying signalling, or the sanctioning techniques introduced above, to applications in data sharing and information processing, the key requirement is to provide meaningful information-related measures in order to evaluate the quality of an interaction.

We can distinguish among objective measures that can be principally verified by all parties involved in a process, and subjective measures that are used by participants and are principally not known to other participants, though they might build hypotheses about them. Examples of objective measures are the price of a product, measurable quality aspects of a data, and the level of privacy maintained when releasing a piece of information. We consider privacy as a measure, since we interpret it as the degree of access to information that can be gained by participants, respectively the maximum information exposure by a participant considering available analysis mechanisms. Examples of subjective measures are trust the degree a participant believes another participants will cooperate, utility and credibility of content the degree a participant believes information is useful or correct.

Since trust mechanisms are inherently feedback systems, they may exhibit complex system dynamics. For some (loosely coupled) systems the dynamics may be described by mean field equations [45], whereas more complex and strongly coupled trust systems may exhibit complex non-linear dynamics. The dynamics of the evolution of trust has also been studied in evolutionary game theory.

Numerous techniques, using cryptographic and inference methods, have been devised to solve specific problems of trust and privacy in Web information systems and many systems are now deployed in practical contexts, one of the best known being the rating mechanisms in eBay. The presence of multiple mechanisms leads immediately to the question of how they can interoperate, since different sources of reputation information might be aggregated to obtain a more complete picture of the trustworthiness of a participant. This requires an interoperability approach that brings today's isolated solutions together [79]. Currently, major players delivering a multiplicity of services: as identity providers, reputation aggregators, service providers and trust evaluators. Establishing a more even power balance might arguably follow a separation of concerns approach.

In order to establish interoperability and separate concerns, semantically interoperable data and services are required for a disaggregated trust and incentive infrastructure to work seamlessly. This is where the web of linked services not just linked data holds promise as a scaleable approach for disaggregated, interoperable brokerage.

\subsubsection{Web of trusted, linked services}

Consider the following scenario illustrating the GPP's use of dynamically configured web services in support of the new forms of enquiry that we envisage:

A virtual team of social scientists, policy advisors, and citizens who have established sufficient reputation from prior experiments, are co-developing a model. Realising that they are missing up to date data, the GPP transforms this into a request for a custom 
app, released to thousands of registered users to participate in the experiment. They download the app, share data from their phones, which is routed back to both the science team and the public, being cleaned, transformed into linked data, and visualised in different ways for different audiences.

The Future Internet is an EU initiative which brings together over 150 projects with a combined budget of over $400 \mathrm{M}$ Euros to create a new global communications infrastructure which can satisfy Europe's economic and societal needs $[23,76]^{3}$. The Internet of Services is a significant layer within the above, providing a technical platform for the Service Economy over new and emerging network infrastructures. Web service technologies are a key technology here since they provide an abstraction layer, through service interfaces (or endpoints), which allow heterogeneous computational components to be accessed via standard web protocols. As such, Web services are widely used within enterprise settings to support the provisioning and consumption of business services.

Recently, Semantic Web technology has been applied to Web services to reduce the effort and resources required to carry out the tasks associated with creating applications from Web service components. Specifically, Web service ontologies have been created, such as the Web Services Modelling Ontology (WSMO) [17] which can be used to describe services in a machine-readable form enabling the semi-automation of service discovery, composition, mediation and invocation. Building on top of service ontologies, such as WSMO, the notion of a semantic service broker e.g. [23] was developed. Semantic service brokers are able to mediate between client requests and service capabilities, describing each part semantically and using reasoning the bridge between the two. Key to this was the use of an epistemology capturing the desires of users around the notion of formally defined Goals which were distinct from service vocabularies and the notion of Mediators to formally describe how semantic and interaction mismatches could be automatically resolved.

For example, using a semantic service broker a scientist could submit a goal to view live traffic data from an environmental impact point of view. Using a goal and service library, a workflow would be configured, combining services for: gaining live traffic information within a region; measuring carbon monoxide, calculating noise and vibration levels; accessing regional fauna and flora data, and visualizing the resulting datasets.

Recent work has led to the emergence of Linked Services [51] which provide a means to place and manage services over Linked Data [8]. As the simplest form of the Semantic Web, Linked Data has recently been take-up by a number of major Media and Web players such as: the $\mathrm{BBC}^{4}$, Google $^{5}$, Facebook ${ }^{6}$, Yahoo! ${ }^{7}$ and Microsoft ${ }^{8}$ as well as a number of national governments ${ }^{9}$. This has led to an emerging the Web of Data, which as of September 2011, was seen to comprise over 31 billion statements ${ }^{10}$. Extending the above notions, Linked Services are services described using Linked Data, consuming and producing Linked Data as input and output. Having a

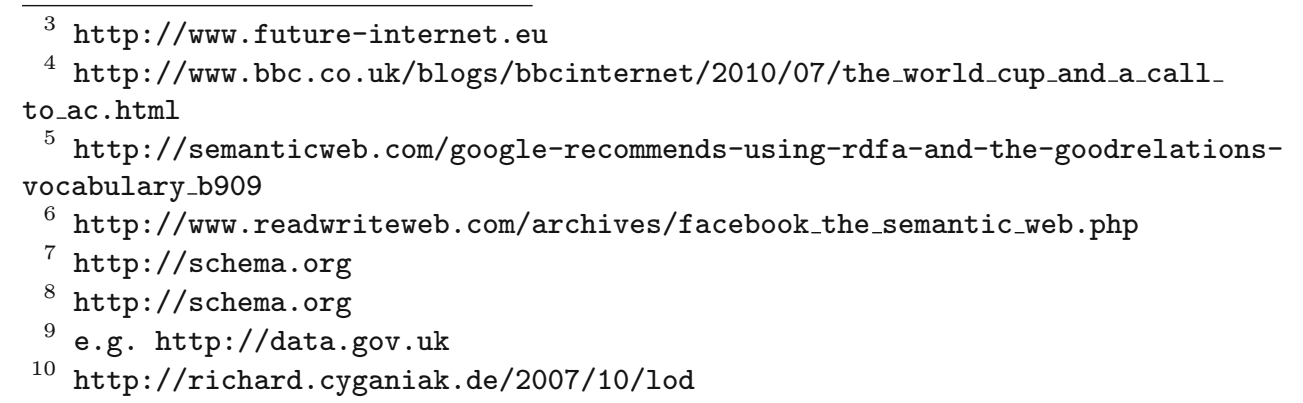


uniform language for both these two roles greatly simplifies the integration of data and functionality, and facilitates automation based upon machine-readability.

\subsection{Collective intelligence}

Many of the problems now confronting us, at all scales, are beyond the capacity of any individual to solve or act upon. Moreover, effective action in complex social systems cannot be effected unilaterally - there is no solution if there is no ownership by and coordination across multiple stakeholders, whether this is a small team, organisation, network, community, city, region or nation. We need breakthroughs in our collective intelligence - our capacity at different scales to make sense of problems, to construct new datasets, analyse them and consider their implications.

In this final review section, we consider some of the issues raised by opening up to wider audiences the interpretation of big data and the models/simulations built on top of them - and inevitably, the debates these will catalyse over the implications for science and policy.

\subsubsection{Citizen science}

There is a long history of successful citizen science. In fields as diverse as astronomy, archaeology and ornithology, amateur scientists have made significant contributions. But the last decade has seen a huge expansion in the sorts of scientific endeavor that non-professionals can contribute to, thanks to the extraordinary development of information technology. It is now possible to play computer games that solve deep challenges in protein folding, simulate the flow of water through nanotubes on a home PC to help in the design of new water filters, or create networks of earthquake detectors using just the motion sensors in laptop computers ${ }^{11}$. We label this new trend citizen cyberscience, to distinguish it from its pre-Internet ancestor.

FuturICT's mission is to help shape the collective computing paradigm, and citizen cyberscience (the form of citizen science that relies on Web infrastructure) embodies this collective computing paradigm in several distinct forms: volunteer computers for sheer processing power, volunteer sensors (typically in the form of mobile phones) for recording data from the real world, and volunteer thinkers, solving problems collectively that can stump even the best professional scientists.

There is a rich ecosystem of citizen cyberscience projects already active today, some involving just a few dozen participants, some hundreds of thousands of volunteers. In total, the number of citizen cyberscientists is well into the millions - no exact data exists, but one of the biggest platforms for volunteer computing, the Berkeley Open Infrastructure for Network Computing (BOINC) counts over 2.2 million users representing $6.6 \mathrm{~m}$ computers. These citizens form the grass-roots core of the global participatory platform envisaged in this paper.

Most of these volunteers are in industrialised countries where there is both Internet access and leisure time to partake in research. But the ubiquity of mobile phones, even in remote regions of the world, is rapidly expanding the opportunities for citizen cyberscience, to even the most seemingly unlikely participants, such as hunter-gatherers in the Congo Basin, a trend which is part of the ambition of extreme citizen science.

The ExCiteS group at UCL is researching existing methodologies, motivations and technologies being used in the full range of citizen cyberscience projects in order to

11 E.g. Quake Catcher Network: http://qcn.stanford.edu 
evaluate methodologies and technologies so that best practice guidelines are established. ExCiteS is also developing new methodologies and technologies in a range of projects from forest communities monitoring illegal logging in Cameroon, to residents of a deprived housing estate in London monitoring noise levels and pollution.

The Citizen Cyberscience Centre (CCC), based at CERN in Geneva, is a partnership promoting the uptake of citizen cyberscience by scientists in developing countries, to tackle urgent humanitarian and development challenges that these countries face. For example, earthquake detection in South East Asia, water filtration in China, deforestation monitoring in Latin America and tracking the spread of AIDS in Southern Africa are examples of the sorts of problems that the CCC is tackling in coalition with local researchers.

Such extreme and practical examples of citizen cyberscience indicate that the GPP can support not just comparatively wealthy and connected citizens, but also inspire innovation and participation of a much wider swathe of the global population. For this to occur, the GPP must provide the tools to both collect, visualise and analyse the data citizen scientists collect in a way that is comprehensible to the many, not just the few. If this goal can be achieved, the GPP would offer the potential to achieve a critical mass of public participation that would assure that scientific creativity goes global, grows exponentially and is supported from within the community of existing users rather than uniquely by professionals.

Through these activities we have found that addressing environmental issues is a major motivator for communities to engage in citizen cyberscience projects. Developing a platform to support communities to address issues of environmental justice is likely to be a major driver of public participation in GPP. Working with international institutions concerned with environmental monitoring and climate change, ExCiteS and the Citizen Cyberscience Centre can offer the GPP the potential to become a platform for storing and analysing data on climate, biodiversity and other critical datasets from all over the world.

Data of the quality required to evaluate climate change at a planetary level is prohibitively expensive if collected only by professional scientists. However, through the intensive mobilisation of citizen scientists, approaches to effectively modelling global climate change patterns and their local impacts become a possibility. To achieve this aim GPP could provide a range of software that allow any community to contribute data from their local area using everyday devices such as smart phones, GPS units or other instruments depending on their objectives, manage the data uploaded (security, permissions etc), run a range of analytical programmes on the data which could show the results in various visualisations that do not necessarily depend on script in order to include the less literate in understanding, analysing and developing action plans based on the data.

As FuturICT has a long term vision to operate on all levels of society and in all parts of the world, we can identify several core research challenges. These include:

- How do we change from a model of passive democratisation to an active one, where we encourage wider groups of participants to see the value in their engagement with FuturrICT products and use it?

- How do we create interfaces and systems that are aimed to facilitate communal cognition, and improve the potential of collective intelligence to foster strong social ties and deliberative processes? To date, systems from Facebook to Wikipedia are suffering from methodological individualism, which is the assumption that instead of dealing with a community as such, they are interacting with each member separately. Yet, we know that the real power behind these systems is in the community aspect. There is, therefore, a need to develop conceptual models and interface that are geared towards this epistemology and view the FuturICT platform as a communal resource, rather than an individual one. 
- How to foster deliberative and inclusionary citizen science process? The current range of recommendation systems, Open Source and citizen cyberscience projects tend to give the voice to those who are the loudest, and exclude (and even alienate) some groups and participants whose views, insights and opinions are silenced.

- How can we integrate everyone including low income, low literacy communities in the most marginal living environments in the collection and use of data sets and models?

A potentially powerful contribution of FuturICT to the creation of sustainable future can be to help small scale farmers in remote part of the world use modelling to improve the outputs of their crop or to enable slum dwellers to understand and improve the utilisation of water resources that are available to them. This may seem far-fetched at present but who would have predicted, just a decade ago, that half the population of Africa would have mobile phones today?

FuturICT should investigate the use and extension of existing platforms for citizen cyberscience to ensure greater inclusiveness and more intense group collaboration, making extreme citizen science more a norm than an exception. There is no doubt that citizen cybercience is a vehicle to engage citizens in a very direct way with scientific research, modeling, analysis and action. However the time has come to move the focus of such projects beyond fundamental science - analysing signals from deep space or folding proteins - and integrate them into the socio-economic, political and environmental concerns of their own personal lives and the places they live in.

The notion of "democratisation" that is frequently used regarding science and the web is more about the potential of the web to make scientific information and modelling accessible to anyone, anywhere and anytime than about advancing the specific concept of democracy. While many use the word to argue that the scientific practice was (and is) the preserve of a small group of experts, and now is potentially accessible to a much larger group, it would be wrong to ignore the fuller meaning of the concept.

Democratisation has a deeper meaning in respect to making scientific data and the practices of its manipulation more accessible to hitherto excluded or marginalised groups. Democratisation evokes ideas about participation, equality, the right to influence decision making, support to individual and group rights, access to resources and opportunities, etc. [24]. Using this stronger interpretation of democratisation reveals the limitation of current practices and opens up the possibility of considering alternative developments of technologies that can indeed be considered as democratising. The dynamics that incentivise participation vary widely, depending on one's conception of citizen science.

To understand the different levels of democratisation that are made available in citizen science, we offer a framework that classifies the level of participation and engagement of participants in citizen science activity. While there is some similarity between Arnstein's [5] 'ladder of participation' and this framework, there is also a significant difference. The main thrust in creating a spectrum of participation is to highlight the power relationships that exist within social processes such as planning and or in participatory mapping [69]. In citizen science, the relationship exists in the form of the gap between professional scientists and the wider public. This is especially true in environmental decision making where there are major gaps between the perceptions of the public and the scientists of each other [5].

In the case of citizen science, the relationships are more complex, as many of the participants respect and appreciate the knowledge of the professional scientists who are leading the project, and can explain how a specific piece of work fits within the wider scientific body of work. At the same time, as volunteers build their own knowledge through engagement in the project, using the resources available on the 


\section{Level 4 'Extreme Citizen Science'}

- Collaborative Science - problem definition, data collection and analysis

Level 3 'Participatory science'

- Participation in problem definition

and data collection

Level 2 'Distributed Intelligence'

- Citizens as basic interpreters

- Volunteered thinking

Level 1 'Crowdsourcing'

- Citizens as sensors

-Volunteered computing

Fig. 3. Four levels of participation and engagement in citizen science.

Web and through the specific project to improve their own understanding, they are more likely to suggest questions and move up the scale of participation.

Therefore, unlike Arnstein's ladder, there should not be a strong value judgment on the position that a specific project takes. At the same time, there are likely benefits in terms of participants' engagement and involvement in the project to try and move to the highest rung that is suitable for the specific project. Thus, we should see this framework as a typology that focuses on the level of participation (Fig. 3).

At the most basic level, participation is limited to the provision of resources, and the cognitive engagement is minimal. Volunteered computing relies on many participants that are engaged at this level, and following Howe [34] this can be termed 'crowd-sourcing', part of the broader conception of collective intelligence being developed here.

The second level is 'distributed intelligence' in which the cognitive ability of the participants is the resource that is being used. The participants are asked to take some basic training, and then collect data or carry out basic interpretation activity. Usually, the training activity includes a test that provides the scientists with an indication of the quality of the work that the participant can carry out. The next level, which is especially relevant in 'community science', is a level of participation in which the problem definition is at least partly shaped by participants, and in consultation with scientists and experts a data collection method is devised. The participants are then engaged in data collection, but require the assistance of experts in analysing and interpreting the results. This method is common in environmental justice cases, and goes towards Alan Irwin's [36] call to have science that matches the needs of citizens.

Finally, collaborative science may become a completely integrated activity, as it is in parts of astronomy, where professional and non-professional scientists play all roles: deciding on which scientific problems to work, the nature of the data collection so it is valid and follows scientific protocols, while matching the motivations and interests of the participants. The participants can choose their level of engagement and can be potentially involved in the analysis and publication or utilisation of results. This form of citizen science can be termed as 'extreme citizen science' (ExCiteS) and requires professional scientists to act as facilitators, in addition to their role as experts. 


\subsubsection{Serious gaming}

With the objective of understanding reciprocal systems, the role of the user within the environment both as participant and researcher opens up new potential for crossdisciplinary and cross-sectoral and trans-age environments, where communities can interact together to solve problems and create group hypotheses, as well as testing existing theories and modelling potential futures. In work being undertaken at the Serious Games Institute, multiplayer environments are being developed in stages which will support cross-disciplinary education for children: the Roma Nova project $[3,50]$. The environment brings together 'gamification' elements with an open virtual environment, supporting coordinated game play (missions and quests) that seek to solve problems and breakdown the separation between formal and informal education, teacher-led and participatory teaching and learning, single disciplinary and multi-disciplinary learning, by combining different interfaces, agent-based scaffolding and supporting social interactive learning. The game allows users to interact with and filter big data on-the-fly, utilise semantic web mash ups according to geocoded spaces and provide a pedagogic underpinning to the serious game design (e.g. [26]).

This existing work and experience in serious gaming provides a springboard for the development of a massive multiplayer online gaming environment to facilitate experimentation and data collection for the GPP. The gaming environment, called the World Game Platform, will be portable to any device, including smart phones and other mobile devices, and integrates new interfaces such as augmented, tactile control, and brain-computer interfaces. This setup allows children to play and learn, testing hypotheses, solving problems and collaborating in social groups in a multilayered gaming environment with high fidelity graphics and realistic game behaviours $[4,52]$. The introduction of artificial intelligence and virtual agents allows capabilities such as data filtering and on the fly analysis but in a synthesised and seamless dynamic system [57]. A mixed-reality connection allows game designers to merge virtual and real-world elements so that games can be intimately connected to the world around us.

This approach will guide the development of the World Game Platform as a FuturICT exemplar project. Here, the participatory design approach will utilize crowd sourcing and distributed computing as in the Foldit project, modelling of quests and missions, geocoding with real world spaces, emergent and dynamic big data analysis and filtering and the adoption of cross-disciplinary and trans age learning could offer the earliest example of a truly reciprocal dynamic gaming system. The interactions of the user model with the game model allow for feedback, optimisation, parameter changing and analysis within the game environment, scaffolded and social interactive learning, and multiplayer engagement and motivation, whilst bringing together complex data filtering and analysis which can facilitate collaborative and community decision making and policy development, scenario planning, emergency management response and evacuation training scenarios in a 'smart cities' modelling and scientific environment as envisaged for the first World Game Platform exemplar.

The main technological challenges here include old issues, such as processing power, low-latency network transmission, and access to technology and levels of innovation in representation. However, when we consider the need to scale and make sustainable systems used by large numbers of users, load bearing, server architectures, cloud computing and large capacity secure storage facilities are all important research and development considerations when addressing issues such as data protection, intellectual property generation and open access. The need to balance between open access, safe storage and recall of information and ethics of intellectual ownership is critical to the success of these reciprocal systems, and creative commons licensing and personal data disclaimers need to be considered in the earliest development stages. 
Technological inclusion is also an important consideration, in particular the ability to reach widest audiences.

It is worth considering current large player base serious online games, such as America's Army and virtual environments, such as Second Life and Club Penguin. While these include millions of distributed players which require dedicated server facilities, the main advantages of these environments are that they engage players for very long periods, a trick that is not always easy to achieve with serious games (games used primarily for non-entertainment purposes) [25]. However these reciprocal systems involve far more complexity in that they tap into databases, as well as user data (sensors), together the data coming from the game play constitutes a huge research challenge: which data do we filter out, what goes back to the user model, what can be shared and how. A user charter is suggested here that makes clear from the outset that this is a research environment, and that certain data (e.g. personal data) will be anonymised and other data made available to the participating research communities.

Alongside this, the research challenge of data retrieval, integration, exchange and analysis a primary question around the use of games, modelling and simulations is one of data presentation and interface. The benefits of using the multiplayer game are in its mode of presentation, which is engaging, provides incentivisation and is easy to use. The game then can be thought of in its widest meaning, as a 'wrapper' for other big data. While this is a relatively under-researched area it neatly brings together the benefits of integrating different models and technologies such as semantic web technologies, mash ups of different applications (and Apps in App Store), geocoding (positionality and sensors in physical spaces), participatory design models (user engagement and collaboration), user and game modelling (integrating high end technologies with Big Data) and social community load bearing and usage (supporting large distributed numbers of participants in science experiment and forecasting), alongside a host of other useful research and research hypotheses generated from the actual simulations and games developed as part of the system.

In this way, a myriad of applications may together be used to model higher level aggregations of data, and analyzed using new tools developed bring together vertiginous links and analyses, processes and behaviours for system refinement and development. The data collected from users (as sensors), within the online game and across the project, may be used to provide sophisticated feedback, and this process itself would have research benefits in terms of predicting human behaviour in certain situations and contexts, calculating next steps with varying degrees of accuracy and modelling spaces where innovation and scientific advances can be best supported in terms of large group interactions and knowledge transfer [15]. Currently there is little or no evidence to understand these interactions in complex online environments save social network analyses. Understanding group and social dynamics therefore is a substantial research challenge.

In serious multiplayer online games, learning of some sort is a desired outcome. While the attraction and retention of users is a priority in gaming, research indicates that if the goal of the game is perceived as serious (for educational or science purposes), it may not attract users for a long period [26]. Therefore, a major research issue is how to design new reciprocal systems that are both engaging and realistic, yet can also teach, and are interactive and retain audience for long periods. The main approach for solving this challenge would appear to be in participatory design approaches to co-design reciprocal systems, in which users are able to co-create, control and utilise live data on-the-fly. Furthermore the system must be sensitive to the users' needs, requirements and history, thereby allowing for increasing challenge for the player to match his or her level of interaction and game play. The main design consideration for this will be in terms of a sophisticated user model that reacts to 
feedback from the game model itself [26]. The user model and the game model will in this way interact in the system allowing game play to adapt to the skills learnt on the part of the user. The design will be a second generation of serious game design that allows for data filtering and processing, user profiling and personalization and game design and modelling all in parallel.

\subsubsection{Information visualisation}

Information visualisation is an interdisciplinary science field that emerged from scientific visualisation. The earlier roots of information visualisation go back to the work of Jacques Bertin, especially his theory of graphics [7]. The philosophy underlying Bertin's work is called semiotics, following the notion that visualisation is more a learned language than a science. Today, this notion is often countered by identifying more and less effective diagrammatic notations mainly based on perceptibility, i.e. we distinguish symbols as more or less effective depending on the way they use the perceptual processing power of the brain without learning [82].

Information visualisation as a research field emerged at the end of the 1980s also from the user interface community [15]. The motivation was to use the advances in graphics hardware for a new generation of user interfaces, especially for interaction with large amounts of information and dynamic queries. The mounting number of different visualisation techniques motivated the work on taxonomies and frameworks to describe the approach of information visualisation and to classify the numerous techniques accordingly. Information visualisation and its related areas, including scientific visualisation, information design, interaction and user interfaces, and cognitive science, are influencing each other, making information visualisation a multidisciplinary field.

Recent developments in the information visualisation community show a possible path ahead for interactive visualisation of distributed data sets. IBM has launched their ManyEyes platform in 2007, a website that allows any user to upload a data set in a certain format and visualise it with various pre-defined visualisation tools online. The users can decide whether they want to share their visualisation, so that other users can comment on them and reuse the findings or even the underlying data. Similar "visualisation tools for the masses", as they were called, were introduced by swivel. com and Gapminder (now owned by Google) around the same time. Swivel no longer exists, mainly because of a lack of support from the visualisation community ${ }^{12}$.

The common idea of these online sites was and is to give any user without special knowledge on how to build and design visualisation tools the means to analyze (relatively simple) data sets. The constraints are the availability of only fixed sets of visualisation techniques, the requirement to upload data in a specific format (certainly a barrier for the lay user) and the relatively restrictive interaction capabilities. It should be noted that while these platforms were relevant and important steps in the right direction, they do not yet provide adequate and sufficient support for GPP.

The VisMaster project [38], a consortium of 26 leading visualisation and visual analytics experts in Europe funded by FET Open, has identified challenges for the visualisation community and published these findings in a European research roadmap. A considerable part of these findings goes exactly into the direction of enhancing the capabilities of visualisation tools "for the masses".

One challenge is the availability of such tools on distributed data sets, which is mainly rooted in the lack of a common platform and interfaces between visualisation tools, development tools, data sets and online apps. This challenge is an obvious goal of the Global Participatory Platform as outlined in this paper.

\footnotetext{
12 http://eagereyes.org/criticism/the-rise-and-fall-of-swivel
} 
For truly democratizing access to complex models and information, the challenges go beyond these general ones. It will be necessary to educate information and tool providers through GPP design guidelines for user interfaces and human-computer interaction based on knowledge about cognitive and perception science.

The GPP will encourage sharing visualisation tools and data sets (based on Linked and Open Data), while ensuring necessary data protection measures to meet privacy concerns. FuturICT envisions a broad availability of a much wider set of visualisations than anything that ManyEyes provided. The visualisation community will highly appreciate such a platform, since the enhanced comparability of visualisation techniques through a common set of data sources is pushing the community forward. The lay user receives the benefit of high-quality, frontier visualisation techniques to use with their data sets, with a much higher trust in FuturICT than in any one of the company-owned websites.

\subsubsection{Argument visualisation}

In the introduction, we framed Collective Intelligence (CI) research as the field investigating the design of infrastructures to enable collectives to act intelligently - and intriguingly, more intelligently than individuals. A particular line of research relevant to the GPP seeks to understand the particular forms of CI that can be constructed through open, reflective discourse, which enables advanced forms of collective sensemaking, such as idea generation and prioritization, deliberation and argumentation.

Online dialogue in conventional social media platforms is unstructured and data is not presented in a way that makes it easy for other people (or machines) to make sense of (or extract) the rich social and technical knowledge embedded in the dialogue. For instance, there is no way to assess the state of a debate: how protagonists are using a given source, who disagrees with whom, or why. Approaches to visualizing argumentation [11] as semantic hypertext networks have been shown to augment sensemaking in diverse contexts where teams are tackling truly complex problems (e.g. participatory urban planning [16]) or NASA lunar exploration [70]. Explicit semantic networks provide a computational system with a more meaningful understanding of the relationships between ideas than natural language. Following the established methodological value of Concept Mapping [46], the mapping of issues, ideas and arguments extends this to make explicit the presence of more than one perspective and the lines of reasoning associated with each. More formal approaches, derived from the convergence of AI and argumentation theory [55,81], model argument structures in finer detail, thus enabling automated evaluation [6]. An ongoing research challenge is to add such computational power without sacrificing usability for non-experts.

A comprehensive review of computer-supported argumentation for learning [65] concluded that studies have demonstrated that the use of argument mapping tools leads to: "more relevant claims and argumen... disagreeing and rebutting other positions more frequently... and engaging in argumentation of a higher formal quality." However, for the GPP to use argument maps as part of its communication and educational strategy (see the Education paper [37]), appropriate tools need to be part of an effective learning design: "The overall pedagogical setup, including sequencing of activities, distributions of roles, instruction on how to use diagramming tools, usage of additional external communication tools, and collaboration design, has an influence on learning outcomes."

Motivated by the challenge of raising the quality of debate, and opening it up via participatory platforms, many research and some business tools are emerging, including the Open University's Compendium [12] and Cohere [13], MIT's Deliberatorium 
[35], Price and Baldwin's Debategraph ${ }^{13}$, and many others ${ }^{14}$. The answers to complex questions of the sort that the GPP aims to support are rarely simple, but their complexity can be managed through argument maps, using visualisation techniques to show clearly how one model might challenge an assumption behind one course of action, how another model predicts that a risk is in fact lower than common sense reasoning envisaged, or how a third model raises new questions, but suggests a course of action which combines two others already considered. These tools are intended for deployment with stakeholders interested in questions such as, What do these three simulations have to contribute collectively to the policy dilemma we face? or Do these three data sets, when combined and loaded into this game, support or undermine this theory's predictions?

Tools such as Cohere provide proxy indicators of participants' attitudes towards the topic under discussion (e.g. someone who disagrees with a particular position), and of the roles they play within the discussion group (e.g. brokers who connect the thinking of peers) [18]. Moreover, the social network structure can be extracted and overlaid on the conceptual network of the discourse. This enables system recommendations that encourage new approaches to a given subject, by providing links to resources that challenge or extend learners' point of view, or by providing links to other groups talking about the same subject or resources but in different ways. Moreover, computational parsing of prose can now detect the salient rhetorical markers used by authors when signaling a knowledge-level claim such as identifying an unresolved problem, or reporting new evidence to support an hypothesis [63] - automated annotation which has now been integrated with human annotation and argument mapping at the Open University. Thus, a new generation of data-intensive learning analytics is emerging, in which the computational platform can gain new levels of 'insight' into the quality of the discourse in order to assist in its moderation, and participant learning or information filtering.

Some of the challenges ${ }^{15}$ for the next generation of computer-supported argumentation platforms, which the GPP will investigate, include:

- Is it possible to host massive online debates, without expensive moderators, and maintain coherence despite intense disagreements and many participants?

- How can a platform proactively support participants in understanding the connections between diverse perspectives?

- What should the next generation of social platforms offer to better detect emergent patterns in online communication?

- Can argumentation platforms gain in computational power and still remain usable by lay people, or are they best seen as power tools for trained analysts to make sense of complex problems?

- Under what conditions do stakeholders networked via argumentation tools outperform individuals, or groups, using conventional collaboration tools?

\subsubsection{Deliberation platforms}

eParticipation is defined as technology-mediated interaction between citizens, the administration and the formal political spheres, usually over some decision-making,

\footnotetext{
13 Debategraph: http://debategraph.org

14 Online Deliberation: Emerging Tools, 2010 workshop: www.olnet.org/odet2010 EScience/Sensemaking/Climate Change tools: http://events.kmi.open.ac.uk/essence/ tools

${ }^{15}$ Collective Intelligence as Community Discourse \& Action, CSCW 2012 workshop: http://events.kmi.open.ac.uk/cscw-ci2012
} 
legislation or simple deliberation process [64]. During the last decade, eParticipation has been a priority for the European Union, giving birth to numerous systems and approaches at EU, national and regional levels, engaging hundreds of researchers and practitioners $^{16}$.

However, although technology is the medium for offering on-line engagement eParticipation services to the public, the real issues are far more important than drawing a technical plan leading to sophisticated computer-supported functionality [84]. Relating to the core objectives of FuturICT, the importance of electronic participation in solving complex societal problems is two-fold: citizens have a way to interact with policy setting, providing their opinion on decisions to be taken, while politicians and policy makers have new means for describing the problems to citizens and then automatically processing their response into meaningful indicators and issues.

The research challenges in this domain, that have been identified by the ICT, complex systems and political science communities over the last 3 years ${ }^{17}$ cover a wide area of multidisciplinary research issues, spanning across the knowledge existing in several FuturICT partners and taking momentum from their collaboration whatsoever.

The key research challenges for the next generation of eParticipatory systems and services align strongly with the services and use cases envisioned for the GPP:

- Transformation of the more traditional eParticipation portals and forums into interoperable services that can reach citizens through a variety of channels. Social media, mobile devices, serious games and other ambient, peer-to-peer technologies need to be properly interconnected in order to multiply the ways that citizen voice can be heard [84].

- Advanced processing tools for extracting knowledge and citizen opinion from typically unstructured, informal inputs. The application of text mining tools, topic-dependent sentiment analysis algorithms and issues extraction mechanisms promise that they can deliver important knowledge towards the further modelling and simulation of complex societal problems [42].

- Further exploration of collaborative governance methods and practices, giving the opportunity to citizens to participate in societal problem-solving and public service co-design, adhering to the Digital Agenda 2020 relevant provisions ${ }^{18}$.

- A new institutional design for collaborative governance, combining ICT capabilities and innovative policy making activities. The combination of digital means (such as text, visualisations, images, video or animation) for the description of policy and the proper setting of such means within the evolving policy-making cycle will be of extreme importance, giving citizens the ability to understand the nature of societal challenges discussed and allowing politicians to elicit and process meaningful results [62].

- Establishment of the foundations of "ICT-enabled Governance" as a new scientific domain, powered by formal methods, metrics and assessment models, decision support, modelling and simulation tools, that aim to support evidence-based policy making with rigorous impact assessment (CROSSROAD project, op cit.)

\footnotetext{
16 E.g. DEMO-NET Project: "The eParticipation Network of Excellence", http://www. demo-net.org, MOMENTUM Project, "Monitoring, Coordinating and Promoting the European Union"

17 CROSSROAD Project: "A participative roadmap for ICT research in Electronic Governance and Policy Modelling", European Commission Support Action, http://www . crossroad-eu.net

18 European Commission: The European Digital Agenda 2010-2020, http://ec.europa. eu/information_society/digital-agenda/index_en.htm
} 
Intersecting with the eParticipation/eDemocracy agenda is a specialised class of participatory platform for argumentation, introduced next.

\subsubsection{Narrative and storytelling}

The GPP seeks to empower ordinary citizens and policy makers alike to access, evaluate, and visualise the ever-increasing amount of data present in our digital world, providing insights that will drive both niche interest groups as well as major policy decisions. Effective communication is a core piece of the GPP's foundation and a necessary component to realise its full promise. However, communicating raw factual conclusions, sterile numeric tables, and lone graphs and visualisations often resonates poorly with audiences, falling flat and leading people to invest their limited attention elsewhere. Moreover, presenting isolated information fails to effectively contextualise it within the reality that we hope to change.

Storytelling provides a proven means to address these shortcomings. As novelist and academic Reynolds Price asserts, "the sound of story is the dominant sound of our lives" [54]. Constructing a narrative around a factual conclusion elevates raw information to the level of insight and connects it to the archetypal human experience to which Price alludes. Narratives can contextualise information in order to highlight the human implications of factual data, connecting it to our shared reality through conflict, character, and plot. Ultimately, storytelling allows us to transform unapproachable scientific data and factual conclusions into the common language that has been used for communication throughout the evolution of the human species.

The structure of the GPP provides a unique opportunity to merge information and storytelling in order to achieve more effective communication. By establishing channels for data contribution and collection, anecdotal information can be included alongside analytics, allowing data mining tools to connect numeric data back to human stories. The communal nature of the GPP allows users to engage one another in discussions about conclusions drawn from individual facets of big data. These shared discussions provide a means for stories to emerge, drawing on the personal experience of the participants. Finally, the collective use of the GPP by groups across the world provides a level of meta-information comprised of the individual conclusions made by each group. Patterns in these conclusions that are evident at a global scale can lead to stories about emergent trends in the world's shifting socioeconomic forces.

Narratology intersects with computing on several research fronts in which FuturICT partners are active:

- Narrative search results: the generation of exploratory interfaces and search results which connect heterogeneous elements meaningfully into a narrative, using semantic templates and natural language generation [44,61];

- The use of narratological models to underpin story-based annotation and browsing: the derivation of a story markup scheme in the design of a prototype 'storybase' for healthcare knowledge sharing [41].

- The distinctive role of narrative as a form of knowledge representation for complex systems thinking: complex systems make sense in retrospect, as analysts seek to construct plausible narratives for each other and decision-makers to make sense of complex systems. Narrative has an important place in some of the most influential work on sensemaking support systems [10]. 


\section{Proposed approach}

\subsection{Participatory layers framework}

"Meaningful engagement" with the concepts and tools of FuturICT raises numerous questions, such as: Who are the users? What are skills and levels of understanding do they bring? What will the project offer them, and what will incentivise them to make use of these resources? What will incentivise them to contribute, and how could this be managed?

Steering clear of implementation details for the present, we propose a framework as a way to structure thinking about these challenges. It is designed to create the conditions for different stakeholders to find their ecological niche, both satisfying their own needs, and contributing to the resilience of the whole system. These niches are conceived as levels within the system, serving the functions of (i) sensing the environment in order to pool data, (ii) mining the resulting data for patterns in order to model the past/present/future, and (iii) sharing and contesting possible interpretations of what those models might mean, and in a policy context, possible decisions. This is summarised in Fig. 4.

Conventionally, scientists and policy makers would be building, analyzing, interpreting, and sharing datasets, plus a select group of software programmers, modelbuilders and citizens. The participatory paradigm disaggregates these functions, and opens each one up to new configurations. The layered framework permits us to talk more precisely about participatory use cases of consumption (left side) and contribution (right side). For example:

- Following the model of Level 4 citizen science (Fig. 3), and the wide range of work on participatory planning and deliberative e-democracy, we aim for citizens, scientists and professional policy analysts to be conceiving new possibilities, learning about the predictions made by models and simulations, drawing conclusions, and debating their implications. At the level of interpreting and decision-making, these stakeholders would be both contributing and consuming:

- Programmers would have particular roles in developing new visualisation tools or mobile applications to help make sense of the models:

- A specific instance of citizen consumption would be school students using appropriately constrained visualisation tools in projects.

- Returning to the earlier discussion of incentives to participate, if citizens had a personal stake in the quality of the data (e.g. public transport decisions will be made based on it), we can envisage them sharing anonymised data from personal devices as they go about their daily lives (such as mode of transport, GPS location, and quality of commute on a given day). Moreover, if they were playing an online game, or tackling a course assignment, they might also be motivated to curate data (e.g. get a spreadsheet working by curating two datasets so that they can be graphed together). They might then use the data themselves to keep their mobile apps as up to date as possible (e.g. is a bus full, or late, or dirty), as well as to ensure that policy makers were making decisions about their lives (such as cutting bus services) using the right data.

\subsection{Participatory spaces and tools}

Given this layered architecture tuned to the needs and contributions of different stakeholders, we now consider some specific (and in some cases overlapping) participatory spaces and tools that could deliver value. 


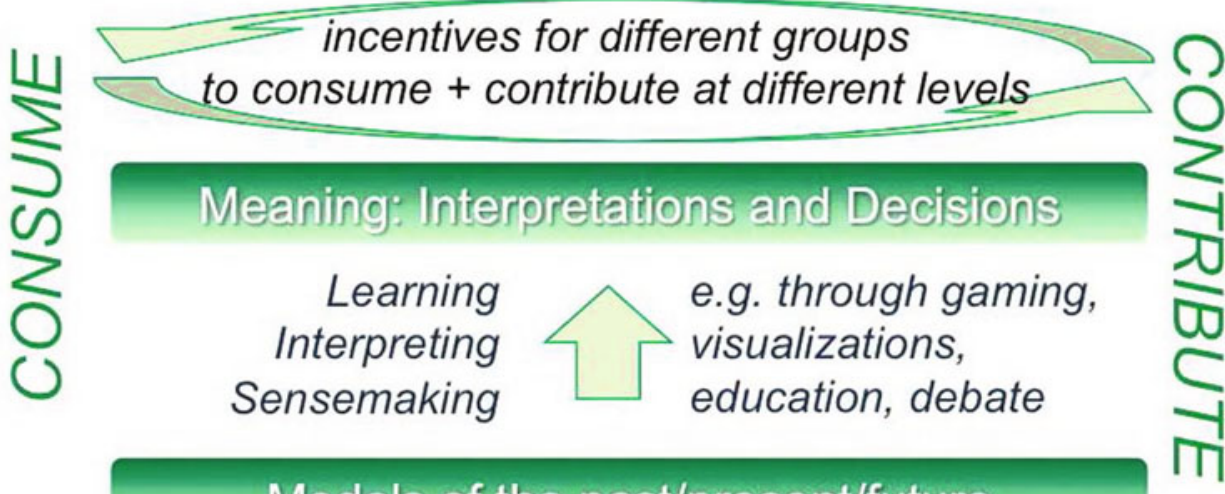

Models of the past/present/future

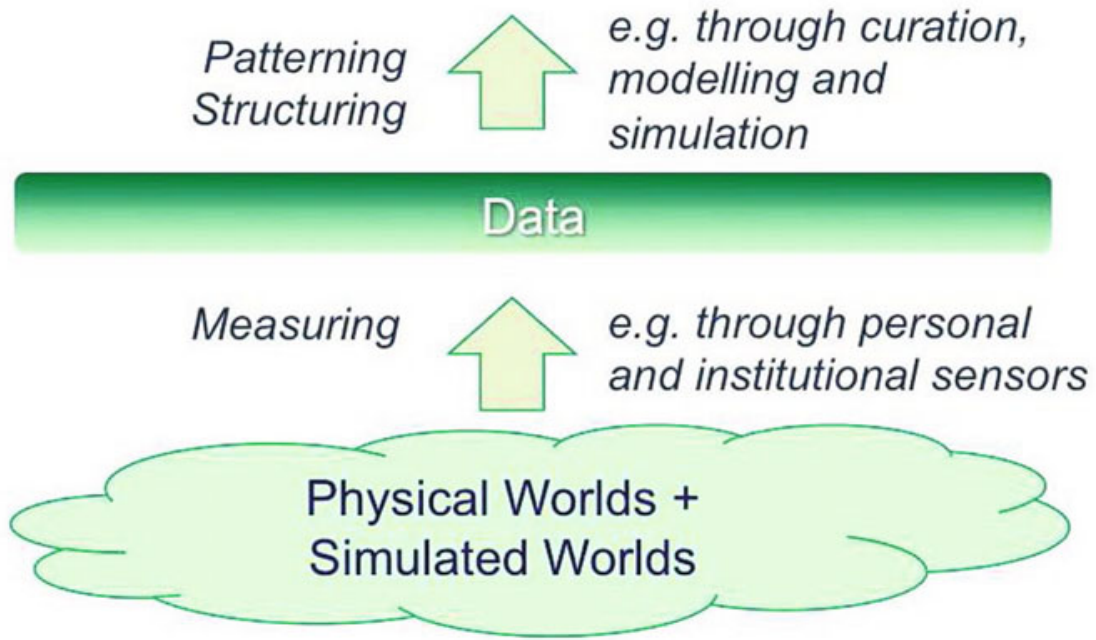

Fig. 4. In the Global Participatory Platform, effective contribution/consumption dynamics will incentivise different stakeholders to participate at different levels in the ecosystem, including data sensing and curation, modelling and simulation, interpretation, debate and decision-making.

An "App Store" providing useful applications running on desktop and mobile devices, which are based on data gathered/generated by FuturICT. Following the app stores/directories we see for iPhone/Android mobile apps, Google Gadgets, Wordpress blogging plugins, etc., FuturICT apps would have to justify their value on their own terms, and would be used through choice by different kinds of stakeholders. They might variously act as sensors to gather contextualised data (e.g. to build an urban heatmap), model-driven advisors (e.g. to plan travel based on traffic models), or games and simulations (e.g. to participate in a contemporary game set in a region, or in a future world in which conditions are rather different, designed to test scientific hypotheses, and/or to educate).

Software developer programme. A good developer programme encourages talented programmers to build on one's platform, tuning it to emergent requirements from communities that cannot be completely envisaged in advance. The platform API co-evolves to meet developers' needs in a symbiotic relationship. The GPP developer programme will provide tools that make it easy to exploit the power of 
the GPP architecture, including developer documentation, tools, code library and news aggregation/dissemination. Examples would include the App Store above, as well as a 'datamart' and 'workbench' providing tools to query, visualise, and download datasets. Initiatives such as Gapminder and DataShop ${ }^{19}$ exemplify the power of shared datasets combined with good analytical tools to make sense of them. FuturICT will deliver similar tools to an eclectic audience, ranging from professional scientists, perhaps all the way to school children, with appropriate supporting documentation and media.

An adaptive online collaboration space connecting people who share common interests, both online and face-to-face. Internet users today are used to a high quality user experience from social networking sites, which they use through choice. The GPP will provide a similar quality of experience, but will quite clearly signpost itself as a place that, while supporting informal interaction, is tuned to building an intentional collective with a common agenda to build deeper understanding of societal dynamics. The next generation of social platforms will make far better use of analytics to provide both users and administrators with pictures of the social networks and communication patterns in the space. A critical requirement will be for recommendation engines to help the different types of user (learners, scientists, policymakers ... ) to navigate the deluge of people, data, documents and claims.

A serious gaming space for exploring and inventing alternative futures. The World Game Platform exemplar will present a visualised and interactive 'smart city' with datasets integrated from economic (e.g. financial markets), environmental (e.g. traffic flow behaviour, energy management and consumption patterns) and social databases (e.g. employment figures, migration and mobility patterns), Big Data from individuals and mobile devices and sensor networks (e.g. positioning, behaviour patterns). The gaming mechanic will allow for players (e.g. citizens, politicians and learners) to participate in city behaviours, decision-making within the environment, research hypothesis, scientific problem solving and social problem testing. The environment will also allow for computer modelling, agent based modelling and simulations to be run within online communities. The WGP will be a platform for communicating scientific outcomes of the project, and for developing new science through mass population testing in the environment. Collective intelligence and distributed computing will provide the bedrock of the environment, and participatory design methodology will be employed to co-create, test and co-design the environment. The smart city environment will allow for more complex modelling approaches bringing together different simulations and approaches within a near 'real world' environment. The WGP will also integrate Apps and Game Apps allowing for ease of use for modelling and testing hypotheses. The WGP will allow for unique methods of communication, learning and research to be generated within the environment. The objectives of the WGP are to allow for more complex and multi-dimensional modelling approaches by utilizing robust reciprocal systems, to test out predicted and unpredicted scenarios and to frame clear research objectives and solutions for adaptation to cascading effects in globalised societies.

A knowledge building space, in which to piece together the insights emerging from FuturICT into different narratives, learning journeys and which maps the intellectual landscapes and intense debates that we expect. Going beyond a collaboration space, the GPP has the key role of communicating the emerging story from FuturICT - answering the question what does all this mean? Part of this will of course be the scientists' stories - their accounts of the implications of their modeling for our understanding of basic phenomena, but also for policy issues. The diversity of models, datasets and scientific disciplines are unlikely to produce a harmonious narrative: it

19 http://www.gapminder.org and http://www.learnlab.org/technologies/datashop 
will be at different levels of analysis, certainly in the earlier phases they will be fragmented, and almost inevitably contradictory and open to debate. As emphasised by the layered architecture, in addition to the scientific narratives, will be interwoven the views of policy analysts, expert groups not formally in the project, and citizen scientists. Tools for building knowledge maps, conducting large scale debates, communicating complex controversies to different target audiences, generating reports, and enabling the tracking of interesting themes will be vital, if the enormous quantity of project outputs is to make an impact.

The above examples do not exhaust the possibilities by any means, but point to ways in which a collective intelligence infrastructure for very large-scale enquiry could orchestrate interaction at different levels.

\subsection{The GPP as a complex social system}

Within FuturICT, a self-reflexive research objective is to apply the concepts and tools of complexity science and social science to the project's own work. We therefore conceive the global participatory platform as a resilient, epistemic ecosystem, whose design will make it capable of self-organization and adaptation to a dynamic environment, and whose structure and contributions are themselves networks of stakeholders, challenges, issues, ideas and arguments whose structure and dynamics can be modelled and analysed. From the perspective of science and technology studies, the categories and models developed, and the representations rendered will themselves be an object of enquiry as we study the way in which they structure interaction between diverse stakeholders.

\subsubsection{The GPP as an open ecosystem}

Contributions to the different levels of the platform, as introduced above, are not only seen metaphorically as the contribution of nodes and edges to an evolving network constituting a distributed ecosystem, but are modelled and implemented as such. Each contribution takes in knowledge and outputs other knowledge, in a way that is similar to, but not identical to, how organisms take in chemicals and excrete other chemicals. Whilst an organism does this to extract energy and other resources from its environment, a person may create and/or maintain a node or configuration of nodes for a variety of reasons, including duty, reputation, profit or altruism. Ecosystems have some desirable properties, including flexibility, robustness, distributedness and efficiency (once adapted) though they are not always rapid.

The key characteristic of an ecosystem (for our purposes) is that it is open in the sense that the output from any node can be used as the inputs for others, so that complex chains of processing can develop, from the simple upwards. The openness of the ecosystem is important for its functioning and flexibility, for if the results of a computation are not effectively available then others cannot invent creative ways of extracting further value from them. To switch metaphors momentarily, a "black hole" node, which would not fit into this model well would absorb huge amounts of data and other input from others, but make it difficult to easily extract it for further processing. This might be represented by a commercial search engine or data aggregator, or a free-loading member of a community who is happy to take but not give back.

Our opening Fig. 1 thus recasts the layered framework as such an ecosystem, taking data streams at the bottom, and through a web of mediating processes, connecting with problem owners at the top who are prepared to input resources into the system in return for possible answers to their questions. Mediating between these is the 
web of software services, plus human networks, curating data, performing analyses, exploring and debating interpretations, and contextualising these to the questions being asked. Thus, there may be services enriching a data stream with location metadata produced by someone/thing else, contextualising the results of a simulation for a particular community, or synthesising answers for a client. Those services might be automated web services, or people such as citizen scientists, government policy analysts, academic researchers, and entrepreneur consultants.

Without committing to implementational details, such an ecosystem would require the following kinds of functionality to be delivered:

- An API for remote services to read, write, and edit nodes;

- One or more portals providing user interfaces for editing, navigating, searching and browsing the knowledge network;

- Service validation to ensure that services comply with the selected open data standard;

- A multidimensional reward system that would allow those agents operating for profit to co-exist with those operating for reputation or other non-financial incentives;

- A way to model and simulate the system as an object of inquiry, including analytics to compare predicted versus observed usage;

- A way to experiment with policies to combat gaming of the system, which might result in a tragedy of the commons (no free-riding).

One instance of a computational system comprising of an "economy of idiots" utilised a distributed ecosystem of problem-solvers to solve "hard" problems using a cascading market-system of rewards [41].

\subsubsection{The GPP as a resilience platform}

A "system", be it a learner, a team, a movement, a network (e.g. social; digital; conceptual), or a city/nation/planet, is considered to be not only sustainable, but resilient, if it has the capability to recover from stresses and shocks, and to adapt its evolution appropriately. Resilience thinking generalises resilience principles from ecology to socio/political and technological systems. Walker, et al. [80] define resilience as "the capacity of a system to absorb disturbance and reorganize while undergoing change so as to still retain essentially the same function, structure, identity, and feedbacks". The FuturICT project's Jamie Macintosh has elaborated the notion further in the context of societal resilience, emphasising that resilience to crises is never about returning to the status quo ante, but always to a new state, and the associated need for the system's transformation: "Resilience is the enduring power of a body or bodies for transformation, renewal and recovery through the flux of interactions and flow of events." ${ }^{20}$ In the context of this project's interest in the impact of ICT on enquiry of all sorts, and the specific education strand, it is noteworthy that it has also established itself in the learning sciences theoretically and empirically as an extremely important disposition, reflecting a learner's perseverance and ability to withstand emotional discomfort and the threat to one's identity, when challenged and stretched beyond one's 'comfort zone' [21] or when confronted by personal and social stressors, often due to poor socio-economic conditions [59].

This section is not entitled the GPP as a resilient system, which would suggest that it should be able to withstand shocks or threats to its infrastructure. While this is obviously desirable, this is not a research focus or design priority, since this is not a

\footnotetext{
${ }^{20}$ UCL Institute for Security \& Resilience Studies: http://www.ucl.ac.uk/isrs
} 
Table 1. Framing the GPP as a resilience platform.

\begin{tabular}{|l|l|}
\hline Resilience thinking principle & Possible GPP as a resilience platform principle \\
\hline $\begin{array}{l}\text { Design diversity into the } \\
\text { system, not out of it }\end{array}$ & $\begin{array}{l}\text { Diversity of participants and viewpoints: design for as } \\
\text { wide a constituency as possible; do not lock participants } \\
\text { into any worldview; support diversity, disagreement } \\
\text { and quality debate }\end{array}$ \\
\hline $\begin{array}{l}\text { Model and implement using } \\
\text { modular components }\end{array}$ & $\begin{array}{l}\text { In contrast to conventional prose, which is opaque } \\
\text { to machine-interpretation, and hard to disaggregate, } \\
\text { model epistemic constructs (such as ideas, questions, } \\
\text { predictions, dilemmas and evidence) as networks } \\
\text { which can grow cumulatively through many autonomous } \\
\text { agents' contributions to an ongoing conversation. }\end{array}$ \\
\hline $\begin{array}{l}\text { Promote practical } \\
\text { experimentation } \\
\text { with feedback loops }\end{array}$ & $\begin{array}{l}\text { Maximise the effectiveness with which agents can } \\
\text { discover new resources or ideas; build in } \\
\text { evaluation/assessment loops }\end{array}$ \\
\hline $\begin{array}{l}\text { Reciprocity in relationships (in } \\
\text { cluding trust and social capital) } \\
\text { is a key resource for negotiating } \\
\text { action under pressure }\end{array}$ & $\begin{array}{l}\text { Recognise and promote the importance of informal } \\
\text { relationships as well as formal, and make use of } \\
\text { appropriate measures of social capital, authority and } \\
\text { reputation. Under pressure, community members will be } \\
\text { more able to rapidly reconfigure, and under stress, call } \\
\text { on the strength of social ties when 'business as usual' } \\
\text { is broken. }\end{array}$ \\
\hline
\end{tabular}

cyber-infrastructure security project. The more interesting sense, for our purposes, is that the GPP could enable a user community to become more resilient: it provides $a$ resilience platform on which they can build with the confidence that it increases their systemic capacity to sense the environment and continue to function when confronted by threats, even reconfiguring under extreme conditions such as a regime shift. More specifically, we are focusing on the knowledge/learning-centric dimensions of such adaptation, since our focus is on improving the analysis of complex questions or dilemmas, in order to inform decision-making.

A key requirement in any complex adaptive system is a degree of self-awareness, through appropriate feedback loops. "Feedback" may be only low-level data signals when we are thinking about biological organisms or digital networks with no human in the loop. However, in a system concerned with higher order cognition, we move from simple positive/negative feedback loops, to epistemic constructs such as ideas, questions, predictions, dilemmas and evidence, and emotional constructs such as surprise, reputation, hope and fear. In other words, feedback/self-awareness implies the capacity to reflect, learn and act effectively, both individually and collectively (cf. the opening definition of Collective Intelligence).

As a preliminary step, we may consider some design principles for resilient systems, and consider with possible translations into principles for a participatory CI infrastructure such as the GPP (Table 1). In considering how a collective responds to overwhelming complexity, a key concept is sensemaking, which has emerged as a definable research field over the last 30 years, dating back to Doug Engelbart's 1960s work (see introduction), and Horst Rittel's formative work in the 1970s on "wicked problems" (reviewed in [11]). As noted in the call for a recent journal issue devoted to the subject [53], influential work has also "emerged quasi-independently in the fields of human-computer interaction [60], organizational science [83], and cognitive science [39]". Browning and Boudès [10] provide a helpful review of the similarities and differences between two influential strands of work on organizational sensemaking by Snowden and Weick, with particular emphasis on the centrality that narrative plays 
Table 2. Sensemaking phenomena in complex domains, and potential roles for the GPP.

\begin{tabular}{|l|l|}
\hline Sensemaking in Complex Domains \\
$\begin{array}{l}\text { Dangers of entrained thinking from experts } \\
\text { who fail to recognise a novel phenomenon }\end{array}$ & $\begin{array}{l}\text { Potential role for GPP } \\
\text { Open up to diverse perspectives }\end{array}$ \\
\hline $\begin{array}{l}\text { Complex systems only seem to make sense } \\
\text { retrospectively: narrative is an appropriately } \\
\text { complex form of knowledge sharing } \\
\text { and reflection for such domains }\end{array}$ & $\begin{array}{l}\text { Coherent pathways are important; Stories } \\
\text { are potent ways to communicate visions of } \\
\text { future possibilities; Reflection and } \\
\text { overlaying of interpretation(s) is critical }\end{array}$ \\
\hline Patterns are emergent & $\begin{array}{l}\text { In addition to top-down, anticipated } \\
\text { patterns, generate views bottom-up from } \\
\text { the data to expose unexpected phenomena }\end{array}$ \\
\hline $\begin{array}{l}\text { Many small signals can build over time into } \\
\text { a significant force/change }\end{array}$ & $\begin{array}{l}\text { Enable individuals to highlight important } \\
\text { events and meaningful connections, } \\
\text { which are then aggregated }\end{array}$ \\
\hline $\begin{array}{l}\text { Much of the relevant knowledge in complex } \\
\text { emergent systems is tacit, shared through } \\
\text { discourse, not formal codifications (Hegel, } \\
\text { et al. 2010) }\end{array}$ & $\begin{array}{l}\text { Scaffold the formation of significant } \\
\text { inter-personal, learning relationships, } \\
\text { through which understanding can be } \\
\text { negotiated flexibly }\end{array}$ \\
\hline
\end{tabular}

in their proposals for how we manage complexity. Table 2 (left column) draws on the key features they, and Hagel, et al. [31] identify, while the right column suggests ways in which the GPP might be shaped in order to tackle some of the breakdowns in individual and personal sensemaking that are known to occur in complex domains. To summarise, we have outlined an approach in which the GPP is itself architected around principles inspired by complex sociotechnical systems, in order to test the hypothesis that we can develop an organic, network-centric, resilience platform for FuturICT as a project, and for other communities to build on.

\subsubsection{The GPP as a boundary infrastructure}

The goal of FuturICT is to provide an innovative framework and components that individuals, communities, enterprises, governments and trans-national organizations can deploy to model and measure human and natural phenomena on an unprecedented scale. The GPP provides the principal means to access the capacity to measure, model and predict. Thus the GPP will become deeply enmeshed within the information infrastructures constructed at a range of scale from the individual to the global. In this context the GPP will deploy, and be supported by, a wide range of knowledge structuring devices such as a classification schemes, data schemes, ontologies, models and simulations, visualisations and reports. These devices are of course human constructions, designed to make the world simpler to measure and hence describe. Our interest in these symbol systems is in how they mediate discourse and, inevitably, conflict, in an open participatory ecosystem of the sort envisaged for the GPP.

Science and technology studies take such devices as principal objects of study, and in particular, Star and Griesemer [75] introduced the notion of "boundary object" to capture the notion of a shared information artifact between two or more communities of practice. Reflecting on the notion of "boundary object" in her posthumous paper, Star [72] summarizes their role:

"Boundary objects are a sort of arrangement that allow different groups to work together without consensus. However, the forms this may take are not arbitrary. They are essentially organic infrastructures that have arisen due to what Jim Griesemer and I called "information needs" in 1989. I would now add "information and work requirements," as perceived locally and by groups who wish to cooperate" (p. 602). 


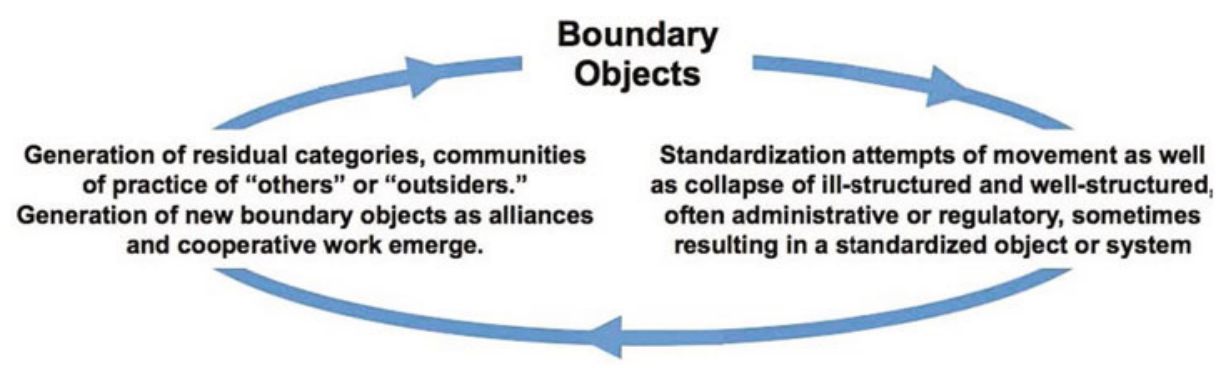

Fig. 5. Boundary objects, standards and residual categories.

Here Star points out a key facet of the notion of a "boundary object" - they allow different groups to work together without the requirement of consensus. Much of the time boundary objects are good enough to achieve effective coordination but local interpretations inside individual communities can diverge at unpredictable moments and require negotiation and exceptional interaction to repair and align interpretations. Later in the same paper Star point out the cycle of continuous elaboration around the invention of boundary objects and their formalization (Fig. 5).

Boundary objects are invented to facilitate working across communities of practice. These are then formalised through a process of standardization or adoption as standard working practices but as this standardization process takes place, new residual categories arise together with new uses for the boundary object and new communities of practice bear on the newly standardizing structure. This then gives rise to new boundary objects and their associated practices that accommodate for the inadequacies of the standardized mechanisms. This cycle repeats, thereby creating layer upon layer of representation and interpretation that facilitates working across communities of practice.

In subsequent work Star and Bowker $[9,73,74]$ develop detailed accounts of boundary infrastructures which "deal in regimes and networks of boundary objects", and document the ways in which information infrastructures are constructed and gradually melt into the background so as to become invisible while still constraining how the field is studied and reported. But these invisible structures potentially provide a focus for radical re-examination of the basis for interactions between communities of practice. When the environment or communities of practice shift they can make previously unimportant failures of consensus critical to the work supported by the Information Infrastructure driving the need for radical re-evaluation of the basis for cooperation. This work from science and technology studies has a critical role to play in the development of the GPP:

- It points to the potential for serious hazards in the deployment of the GPP. If the design of the GPP does not leave the capacity for some "margin for maneuver" once a boundary object has undergone standardization and adoption then the communities of practice may have no means to respond to shifts in circumstances. This could result in extreme difficulty in reestablishing the means for cooperation in the absence of consensus.

- The GPP has the potential radically to alter the instruments that social science has available to study features like boundary objects. The GPP could help provide much deeper understanding of how interacting communities of practice develop and utilize these elements in information infrastructures. Scientific advance in our understanding of these kinds of features has huge potential radically to alter our approach to the design and implementation of critical infrastructures which for 
the most part are underpinned by an information infrastructure that supports

interacting communities of practice.

The study of Information Infrastructure is but one of many areas where there is the potential for synergy between the FuturICT programme and Social Science. The objects of study of Social Science have huge potential consequences for the development of FuturICT, and at the same time, FuturICT has the potential greatly to accelerate progress in some areas of Social Science.

\subsection{Options and tradeoffs}

As we have developed the ideas in this paper within the project, and engaged external audiences, a number of issues recur as points for debate. Over time, they reveal themselves to be important dimensions describing a design space of possibilities for realising the GPP concept, within which choices must be made. As a scientific project, our task is to clarify the choices being made in order to reveal other options for exploration, and study the resulting tradeoffs.

We highlight some of these dimensions here in the hope that it moves the dialogue forward:

- Big Observatories vs. long-tail observatories: designing for everyone

- Collaboration vs. conflict: designing for tension

- Scientific demonstrator vs. production service: GPP.org or .com?

- Safe-fail vs. fail-safe: design metaphors for emergence

- Designing to principles vs net-neutrality: how to design ethical ICT?

\subsubsection{Big observatories vs. long-tail observatories: Designing for everyone}

Through its strategic partnerships, the project has prioritised the development of a number of interconnected, thematic Exploratories (Society, Economy, Technology, and Environment) within which more targetted Observatories are run (e.g. of Financial and Economic Systems, of Conflicts and Wars, of Social Well-Being, of Health Risks, of Transportation and Logistics). As the project works on demonstrators for each of these communities, these will serve as drivers for the development of the technology platforms, which over time will clarify which are the most strategically important generic functionalities and abstract models to develop, in order to make it as easy as possible to launch new Observatories which simply customise the platform to their needs. Some of these Observatories will work with governments and companies, on some occasions around proprietary data, and may investigate user interaction paradigms not accessible to most people, such as very large displays and immersive visualisation domes. We might call these the Big Observatories, since they receive direct funding, and have available to them teams of professional scientists, industry analysts, and government policymakers.

As emphasised in this paper, however, we have an equal priority to democratise such facilities, exploiting the characteristics of the participatory, social web, to create what we might call Long-Tail Observatories (see [2] for details of the long tail). The three opening user scenarios exemplified the idea of empowering niche communities with state of the art tools to build their own Observatories, tuned to their very specific interests - and arguably, providing quality datasets and expertise networks that could not be constructed in any other way. It is unlikely that any other Observatory could service requests about global cat behaviour quite like the Cats+Tremors network. A national health analyst pondering the H1N1 threat might learn a lot from 
the Little Village observatory, based as it is on validated models and datasets, and distilled in argument maps.

What becomes clear, however, is that these are not mutually exclusive categories. While long-tail observatories will extend dramatically the range of topics that can be studied beyond those prioritised in the big observatories, we anticipate that the latter will be unable to do their work without the former. Big observatories will in many cases not be able to acquire quality, local, timely data without the participatory orchestration of many local sensors. But beyond this familiar concept, we envisage that many niche observatories may be choose to participate more meaningfully in the experiments of big observatories, and add far more value than just their sensor data. We move, therefore, towards extreme citizen science as introduced earlier.

\subsubsection{Collaboration vs. conflict: Designing for tension}

In the discourse that dominates much of the rhetoric around Web 2.0, user-generated content, crowdsourcing, collaboration tools and e-democracy, there is an assumption that individuals are essentially well-meaning, seeking common ground, and striving for mutually acceptable ways forward in the complex dilemmas now confronting us. As reviewed in this collection of papers and in popularized accounts [67] there are many well documented, inspiring examples of technologically-amplified cooperative, creative behaviour which have impacts far greater than might have been imagined possible before the social web established itself.

Balancing this, however, is the reality that once the GPP is deployed in serious contexts, in which financial, health, technological or environmental decisions will impact many lives, there will be competing agendas and vested interests in play. An intriguing challenge for the GPP is, therefore, to understand what it means to design for tension and disagreement from the start, and at all levels in the infrastructure. It is possible to point to some examples of what this might mean, but much remains to be done. At the level of internet architecture design, Schutz [66] considers Future Internet applications of Clark's design for tussle principle, which defines a tussle as an "ongoing contention among parties with conflicting interests". Interestingly, Schütz notes:

"Clark recognizes that tussles are not necessarily negative. Instead, they are needed to allow evolution and progress. There is no "final outcome", no "stable point". We, as the architects and engineers, have to understand the rules that define the tussles in order to shape the architecture and to ensure evolvability."

We seek to apply this principle at all levels in the GPP layered framework. To take another example, the objective to construct participatory spaces for computersupported deliberation and argumentation at the top layer of the framework assumes from the start that there will be challenges to the quality of data, assumptions in models, and interpretations of their implications for science, and policy. The concept of contested collective intelligence is already under development by FuturICT investigators [19], as is scientific publishing infrastructure which treats scientific truth claims as plausibility narratives, legitimated by specific norms, and signaled in texts by distinctive rhetorical patterns which are computationally tractable [20].

\subsubsection{Scientific demonstrator vs. production service: GPP.org or. com?}

FuturICT is first and foremost a scientific and technology research enterprise: using technology to learn more about society, which requires a co-development of our understanding in how to design and engineer these new tools - tools which must spread 
beyond the traditional scientific stakeholders. As a publicly funded scientific enterprise, FuturICT's emphasis is on foundational questions, which may lead it to focus on challenges that will not necessarily be addressed by commercial companies, and to focus on ICT that can create social, economic and societal benefits. As emphasised at the start, however, the GPP seeks to provide a new kind of commons in which non-commercial and commercial stakeholders can be of mutual benefit. The GPP provides a vehicle to investigate, and hopefully demonstrate, the new possibilities that are opening up, but a continual challenge will be to balance research demonstration with production service.

A dilemma faced by all researchers studying large scale participatory social platforms is that in order to study next generation capabilities, the platforms must be of sufficient quality (e.g. usable, robust, and with the right cost-benefit tradeoff) that communities genuinely choose to use them, and long enough to evidence authentic usage. Compared to other, older forms of human-computer interaction research (which might require a single instance of a prototype running on one high end machine in a lab) it is neither quick nor cheap to create and maintain useful social web platforms in an agile manner, which can run continuously on diverse platforms, with competitor platforms launching every quarter, such is the intensity of innovation in certain fields. The platform has to approach a state of completion much closer to a production environment, which entails a level of attention to user experience and software engineering that researchers often do not plan for.

Two strategies are to "reuse where possible, invent where necessary" in order to maintain responsive agility long enough to test ideas meaningfully, and to explore creative partnerships with businesses. These strategies apply at all levels of the layered framework, from sensor networks, data hosting and computational processing, to model-building and visualisations, to user experience, social networking and participatory deliberation tools. Thus, we envisage companies identifying vertical markets and solutions within the broad, horizontal space of FuturICT, developing for instance, websites/apps/consulting which use the open datasets and simulation tools to meet clients' needs. We also envisage building on open source platforms that have already been through extensive debugging and evolution, rather than re-inventing those wheels. Project partners are leaders in their respective fields, able to assess critically from first-hand experience the technology maturity levels of candidate technologies.

\subsubsection{Safe-fail vs. fail-safe: Design metaphors for emergence}

Complex distributed systems are not predictable, since what results is at least partially emergent. As soon as people are treated as part of the system, the situation becomes more complex for at least four reasons: (i) people are not stable, predictable actors (although of course at a macro level patterns do emerge); (ii) people adapt available technological tools in creative ways to unforeseen ends; (iii) when people start to interact through a new medium, new modes or versions of organisation can emerge; (iv) as a tool becomes popular and relied upon, assuming the developers are interested in maintaining the tool's relevance, users begin to shape its development.

It is, therefore, with confidence that we assert that the GPP will see failures as well as successes. The ambition of building a fail-safe platform for all stakeholders in a complex network of networks is fruitless, but the ambition of building a platform that is safe-fail [71] seems entirely appropriate, if this is taken to mean a platform whose successes and failures one uses to build one's own understanding of a complex domain, which provides continual feedback to researchers, designers and users/co-designers, and which seeks to build a user community's resilience (cf. Sect. 3.3.2). 
The design stance required to evolve humans and tools must therefore concentrate at least as much effort on post-implementation monitoring, maintenance and adaptation. Metaphors inspired by the human shaping of natural systems such as gardening, farming, cultivating and nurturing seem appropriate in this context, and seem to be supported by the evidence of the most successful social web applications to date, whose state of 'perpetual beta' reflects these metaphors of continuous, living adjustment as new patterns are recognised. A hybrid mix of design methods from human-centred informatics research coupled with those tried and tested in industry most closely fit this conception. It also follows that one of the most promising research trajectories for the GPP will concern theories and technologies which amplify our capacity to detect significant patterns in the usage of the platform. Interaction analytics that rise above low level phenomena and reveal higher level forms of social interaction and meaning-making will therefore be of first order importance, for instance, revealing the structure and dynamics of epistemic collectives (i.e. groups with explicit interest in pursuing an enquiry), phenomena signaling the presence of deep learning, or the reporting of gaps in knowledge and new findings that surprise, confirm or challenge accepted knowledge.

\subsubsection{Designing to principles vs. net-neutrality: How to design ethical ICT?}

Any medium, digital or otherwise, imposes constraints on its users: it structures interaction by facilitating certain forms of activity around and through it, and discourages or makes impossible others. It is always desirable to design with as sound an understanding as possible of the impact that an artifact/system will have, and different disciplines in the project will be sources of insight on how to design for different use contexts.

An interesting debate is emerging around the extent to which we should, or even can, articulate participatory, community-dynamics principles that the GPP should embody and promote, or to what extent, like the original internet protocol, the GPP should embody a version of 'net neutrality' as the over-arching principle, making as few ontological commitments as possible. The question is what is the best approach to avoid a 'tragedy of the commons' (see Sect. 2.1.3), and other forms of abuse such as data pollution, cybercrime and privacy violations? The project subscribes quite explicitly to such values, as would most citizens.

One approach is that the GPP can and should, through its technical design and the practices it encourages, embody and enforce principles based on what is currently known about the creation of sustainable and resilient ecosystems, together with other principles inspired from the ways in which we construct our societal norms. In this line of argument, politicians and the public will welcome the creation of explicitly "ethical ICT", based on value-sensitive design (e.g. privacy by design), in which the goal is to create a market based on European legal and ethical values. This approach to the promotion of ethical values emphasises the need to develop and disseminate a suitable set of social norms for the sustainable use of the system, and to implement a legal framework which allows for effective prosecution for significant misuse of the system.

A different emphasis argues that we do not yet know enough to design from first principles in this way, and moreover, that in attempting to do so, the GPP could inadvertently constrict innovation - the very emergent phenomena that is so important in sociotechnical systems. According to this emphasis, care must be taken in attempting to transplant social norms from one context to new emergent contexts. While such questions may be debated philosophically, FuturICT also provides the opportunity to explore such questions empirically, at scale, as we work with myriad communities 
who will elect (or possibly be required, e.g. students on courses or participants in experiments) to use differently structured participation spaces with different systemic properties.

In summary, while the European and Universal Human Rights declarations serve as our moral starting points, abstract claims about ensuring "privacy", "justice" and "human dignity" must be translated into technical design implications, or remain gratuitous, hence the technical nature of the literature reviews in this paper. We believe that the key principle is to find ways to systematically, meaningfully and usefully address issues around the values being embodied in ICT systems, clarifying the ways in which certain values translate at all levels of the infrastructure. The ethics paper in this special issue explores these issues in depth.

\section{Envisaged impact and paradigm shift}

To conclude, the goal of the GPP is to extend the boundaries of our bounded rationality by providing tools that link human decision making to data and inference at all scales in society. We envisage significant impact at many levels:

- Globally: the power and flexibility of the GPP will make it the natural tool for a wide range of global non-profit organizations tasked to manage a range of exceptional circumstances. For example, health authorities could deploy the GPP as a key tool in pandemic control because it has the capacity both to work with historic, curated, datasets as well as with live, potentially partial and poor quality data supplied by partner organizations, national health services and individuals.

- Government: a key appeal of the GPP to governments lies in the flexibility, timeliness and transparency of testing ideas in a 'policy windtunnel' that contrary to normal practice, uses an open platform to harness unprecedented computational power with unprecedented human collective intelligence. In many contexts, most of the necessary data can be made available to all, and public interest groups will be able to judge the quality of government decision making by exploring and refining government models, with a far greater capacity than any government department can muster, to consider alternate modelling techniques, and critically, alternate assumptions.

- Enterprises: FuturICT could link with large scale enterprises on a not-for-profit basis to use the data and modeling capacities of FuturICT to pilot new approaches to products and services that could be protected from the normal approach to IP protection in order to find more innovative syntheses of approaches taken by different companies and governments.

- Communities: small-scale communities stand to benefit considerably from this approach. For example many rural communities explicitly try to plan housing comprehensively and are concerned to ensure the sustainability of housing over the long term. Access to modeling in the form of the GPP could help local community groups plan effectively for long term trends in fuel prices together with the impact of energy saving schemes on the environment.

- Education: Learners from primary school to retirement homes will have the capacity to connect to contemporary data, configure/customise models, and interpret the results through appropriate visualisations. This has massive potential as a learning tool to allow learners to form a clear view of the consequences of local and global decision taking.

- Innovators and Entrepreneurs: a key part of new enterprise generation based on potentially innovative ideas is to understand the structure and dynamics of markets for new products and to understand the option for manufacture or realization of goods or services. The GPP will enable startups and spinouts to gain 
accurate modelling of potential markets together with tools to explore business options in detail. This is a key part of the Innovation Accelerator that will deploy the GPP to provide access to the power of FuturICT to decision takers in Small and Medium-size Enterprises. We envisage SMEs using the GPP to allow them to embed modeling into products they intend to market while imposing as lightweight an IP regime as possible.

- Business Angels and Venture Capitalists: Angels and VCs take a calculated risk in funding small companies. When the GPP makes FuturICT's simulation and close to real-time data capacities are made accessible in appropriate forms, these could help make those calculations more accurate, thereby reducing the levels of risk by allowing funders to study different business plans combined with different market projections to see how robust a particular startups proposals are.

In conclusion, our vision of the GPP is as an open-access gateway to a data, modelling and sensemaking commons, grounded in a European ethical orientation. In a world where the spaces we believed would remain commons are becoming increasingly "enclosed" by commercial and political interests, FuturICT will retain a stance of truly open and transparent access to this vital new resource for generating and testing evidence to support science and scholarship, civic policy and business investment.

The paradigm shift for which we are designing the GPP centres on the possibility that computational models of societal phenomena, and applications that exploit and render them for different audiences, will come to be embedded within our everyday lives. They will transform not only the work practices of professional scientists and policy analysts, but in addition, the growing numbers of 'serious amateurs', who are in many cases the most authoritative sources of knowledge in their local contexts. Moreover, since this embedding should not be by stealth, but open and participatory, a distinctive feature as this transition takes place will be the growth in societal literacy - the collective intelligence to handle these new, powerful tools for reading and writing meaning. The concept of citizenship will evolve to include the motivation and skills to shape this infrastructure, and in so doing, shape society.

The publication of this work was partially supported by the European Union's Seventh Framework Programme (FP7/2007-2013) under grant agreement No. 284709, a Coordination and Support Action in the Information and Communication Technologies activity area ('FuturICT' FET Flagship Pilot Project).

\section{References}

1. K. Aberer, S. Sathe, D. Chakraborty, A. Martinoli, G. Barrenetxea, B. Faltings, L. Thiele, OpenSense: Open Community Driven Sensing of Environment, Nov. 2 (2010)

2. C. Anderson. The Long Tail: Why the Future of Business Is Selling Less of More (Hyperion Books, 2006)

3. E.F. Anderson, L. McLoughlin, F. Liarokapis, C. Peters, P. Petridis, S. de Freitas, Serious Games in Cultural Heritage, 22-25 September (2009)

4. S. Arnab, P. Petridis, I. Dunwell S. de Freitas, Enhancing learning in distributed virtual worlds through touch: a browser- based architecture for haptic interaction. (Springer Verlag, London, 2011)

5. S.R. Arnstein, J. Amer. Inst. Planners 35, 216 (1969)

6. K. Atkinson, T. Bench-Capon, P. McBurney, Artificial Intelligence Law (Special Issue on eDemocracy) 14, 261 (2006)

7. J. Bertin, Semiology of Graphics: Diagrams, Networks, Maps (University of Wisconsin Press, Madison, WI, 1983)

8. C. Bizer, T. Heath, T. Berners-Lee, Int. J. Semantic Web Inf. Syst. 5, 1 (2009) 
9. G.C. Bowker, S.L. Star, Building Information Infrastructures for Social Worlds - The Role of Classifications and Standards. In Community Computing and Support Systems, Social Interaction in Networked Communities [the book is based on the Kyoto Meeting on Social Interaction and Communityware, held in Kyoto, Japan, in June 1998] (SpringerVerlag), p. 231

10. L. Browning, T. Boudès, Emerg. Complex. Organiz. 7, 35 (2005)

11. S. Buckingham Shum, The Roots of Computer-Supported Argument Visualization (Springer-Verlag, London, 2003), p. 3

12. S. Buckingham Shum, A.M. Selvin, M. Sierhuis, J. Conklin, C.B. Haley, B. Nuseibeh, Hypermedia support for argumentation-based rationale: 15 years on from gIBIS and QOC (Springer-Verlag, Berlin, 2006), p. 111

13. S. Buckingham Shum, Cohere: Towards Web 2.0 Argumentation In Proceedings of the 2nd International Conference on Computational Models of Argument (2008)

14. A.T. Campbell, S.B. Eisenman, N.D. Lane, E. Miluzzo, R.A. Peterson, H. Lu, X. Zheng, M. Musolesi, K. Fodor, Gahng-Seop Ah, The Rise of People-Centric Sensing, IEEE Internet Computing, July/August 12-21 (2008)

15. S.K. Card, J.D. Mackinlay, B. Shneiderman, Readings in Information Visualisation Using Vision to Think (Morgan Kaufman Publishers, San Francisco, CA, 1999)

16. P. Culmsee, K. Awati, The Heretic's Guide to Best Practices: The Reality of Managing Wicked Problems in Organisations (iUniverse Inc., Bloomington IN, 2011)

17. D. Fensel, H. Lausen, A. Polleres, J. Bruijn, M. Stollberg, D. Roman, J. Domingue, Enabling Semantic Web Services: The Web Service Modelling Ontology (Springer, Berlin, 2006)

18. A. De Liddo, S. Buckingham Shum, I. Quinto, M. Bachler, L. Cannavacciuolo, DiscourseCentric Learning Analytics, 27 Feb.-1 Mar. (2011)

19. A. De Liddo, Á. Sándor, S. Buckingham Shum, Comp. Supp. Cooperative Work 21, 417 (2012)

20. A de Waard, S. Buckingham Shum, A. Carusi, J. Park, M. Samwald, Á. Sándor, Hypotheses, Evidence and Relationships: The HypER Approach for Representing Scientific Knowledge Claims, 26 Oct. (2009)

21. R. Deakin Crick, P. Broadfoot, G. Claxton, Assess. Education: Princ., Policy Pract. 11, $248(2004)$

22. C. Dellarocas, Reputation Mechanisms (Elsevier Publishing, 2006)

23. J. Domingue, L. Cabral, S. Galizia, V. Tanasescu, A. Gugliotta, B. Norton, C. Pedrinaci, J. Web Semantics 6, 109 (2008)

24. G. Doppelt, Democracy and Technology (State University of New York Press, Albany, NY, 2006), p. 85

25. I. Dunwell, S. Christmas, S. de Freitas, Code of Everand: Evaluation of the Game (Department of Transport, UK, London, 2011)

26. I. Dunwell S. de Freitas, Four-dimensional consideration of feedback in serious games (Continuum Publishing, 2011)

27. N. Eagle, A. Pentland, Pers. Ubiquitous Comp. 10, 255 (2006)

28. E.L. Eisenstein, The printing press as an agent of change: communications and cultural transformations in early-modern Europe, vol. 1 (1979)

29. D.C. Engelbart, Augmenting human intellect: A conceptual framework, Technical Report SRI Project No. 3578, Summary Report AFOSR-3233, Stanford Research Institute (1962)

30. A. Ferscha, Implicit Interaction (2011)

31. J. Hagel, J. Seely Brown, L. Davison, The Power of Pull: How Small Moves, Smartly Made, Can Set Big Things in Motion (Basic Books, 2010)

32. D. Helbing, S. Balietti, S. Bishop, P. Lukowicz, Eur. Phys. J. Special Topics 195, 165 (2011)

33. C. Hess, E. Ostrom, Understanding Knowledge as a Commons (MIT Press, 2007)

34. J. Howe, The Rise of Crowdsourcing, Wired Magazine, June (2006)

35. L. Iandoli, M. Klein, G. Zollo, Int. J. Decision Support Syst. Technol. 1, 69 (2009) 
36. A. Irwin, Citizen Science (Routledge, London, 1995)

37. J. Johnson, S. Buckingham Shum, A. Willis, S. Bishop, T. Zamenopoulos, S. Swithenby, R. MacKay, Y. Merali, A. Lorincz, C. Costea, P. Bourgine, J. Louca, A. Kapenieks, P. Kelley, S. Caird, J. Bromley, R. Deakin Crick, C. Goldspink, P. Collet, A. Carbone, D. Helbing, Eur. Phys. J. Special Topics 214, 215 (2012)

38. D.A. Keim, J. Kohlhammer, G. Ellis, F. Mansmann, Mastering the Information Age Solving Problems with Visual Analytics, Eurographics Association (2010)

39. G. Klein, B. Moon, R.F. Hoffman, IEEE Intell. Syst. 21, 88 (2006)

40. A. Krause, E. Horvitz, A. Kansal, F. Zhao, Toward Community Sensing, April 22-24 (2008)

41. J. Kwiat, From Aristotle to Gabriel: A Summary of the Narratology Literature for Story Technologies. Technical report, Technical Report KMI-08-01, Knowledge Media Institute, The Open University, UK (2008)

42. M. Maragoudakis, E. Loukis, Y. Charalabidis, A Review of Opinion Mining Methods for Analyzing Citizens' Contributions in Public Policy Debate, August 29 - September $1(2011)$

43. E. Morozov, The Net Delusion. Allen Lane (2011)

44. P. Mulholland, T. Collins, Z. Zdrahal, Story fountain: intelligent support for story research and exploration (2004)

45. J. Mundinger, J.-Y. Le Boudec, Performance Evaluation 65, 212 (2008)

46. J.D. Novak, Learning, creating, and using knowledge: concept maps as facilitative tools in schools and corporations. Lawrence Erlbaum Associates, Mahwah, NJ (1998)

47. W.J. Ong, Orality and Literacy: The Technologizing of the Word (Methuen, London, 1982)

48. N. Oreskes, E. Conway, Merchants of Doubt (Bloomsbury, 2010)

49. E. Ostrom, Governing the Commons (CUP, 1990)

50. D. Panzoli, C. Peters, I. Dunwell, S. Sanchez, P. Petridis, A. Protopsaltis, V. Scesa, S. de Freitas, Levels of Interaction: A User-Guided Experience in Large-Scale Virtual Environments (2010)

51. C. Pedrinaci J. Domingue, Toward the Next Wave of Services: Linked Services for the Web of Data, Journal of Universal Computer Science (2010)

52. P. Petridis, I. Dunwell, S. Arnab, S. de Freitas, Building Social Communities around Alternate Reality Games, 4-6 May (2011)

53. P. Pirolli, D.M. Russell, Human-Computer Inter. 26, 1 (2011)

54. R. Price, A Palpable God: Thirty Stories Translated from the Bible With an Essay on the Origins and Life of Narrative (Atheneum, 1978)

55. I. Rahwan, F. Zablith, C. Reed, Artificial Intell. 171, 897 (2007)

56. J. Rawls, A Theory of Justice (Cambridge Mass., Harvard University Press, 1971)

57. G. Rebolledo-Mendez, D. Burden, S. de Freitas, A Model of Motivation for VirtualWorlds Avatars (Springer-Verlag, Berlin, Heidelberg, 2008), p. 535

58. C. Reynolds, R. Picard, Affective Sensors, Privacy, and Ethical Contracts, In Proceedings of CHI (2004), p. 1103

59. Y. Roberts, Grit: The skills for success and how they are grown. Technical report (The Young Foundation, 2009)

60. M.D. Russell, J.M. Stefik, P. Pirolli, S.K. Card, The Cost Structure of Sensemaking (1993)

61. L. Rutledge, M. Alberink, R. Brussee, S. Pokraev, W. van Dieten, M. Veenstra, Finding the story: broader applicability of semantics and discourse for hypermedia generation (2003)

62. S. Koussouris, Y. Charalabidis, D. Askounis, Trans. Gov. People, Proc. Policy 5, 8 (2011)

63. Á. Sándor, A. Vorndran, Extracting relevant messages from social science research papers for improving retevance of retrieval, 10-14 May (2010)

64. C. Sanford, J. Rose, Int. J. Inf. Manag. 27, 406 (2007)

65. O Scheuer, N Loll, F Pinkwart, B.M. McLaren, Int. J. Comp.-Supp. Collab. Argumentation 5, 43 (2010) 
66. S. Schütz, Lessons in 'Designing for Tussle' from Case Studies, Technical report, Trilogy EU FP7 Project, Deliverable D2 (2008)

67. C. Shirky, Here Comes Everybody: How Change Happens When People Come Together (Allen Lane, 2008)

68. R. Shokri, T. Georgios, J.-Y. Le Boudec, J.-P. Hubaux, Quantifying Location Privacy, May 22-25 (2011)

69. R. Sieber, Ann. Amer. Asso. Geograph. 96, 491 (2006)

70. M. Sierhuis, S. Buckingham Shum, Human-agent knowledge cartography for e-science: NASA field trials at the Mars Desert Research Station, Springer (Advanced Information and Knowledge Processing Series), London (2008), p. 287

71. D. Snowden, Naturalizing Sensemaking (Psychology Press, 2010)

72. L.S. Star, Sci. Technol. Hum. Val. 35, 601 (2010)

73. S.L. Star, G.C. Bowker, How to infrastructure (2006)

74. S.L. Star, K. Ruhleder, Inf. Syst. Res. 7, 111 (1996)

75. S.L. Star, J.R. Griesemer, Social Stud. Sci. 19, 387 (1989)

76. G. Tselentis, J. Domingue, A. Galis, A. Gavras, D. Hausheer, S. Krco, V. Lotz, T. Zahariadis, Towards the Future Internet - A European Research Perspective (IOS Press, 2009)

77. J. Van den Hoven, D. Helbing, D. Pedreschi, J. Domingo-Ferrer, F. Gianotti, M. Christen, Eur. Phys. J. Special Topics 214, 153 (2012)

78. H. Le Vu, K. Aberer, Effective Usage of Computational Trust Models in Rational Environments. ACM Transactions on Autonomous and Adaptive Systems (2011)

79. H. Le Vu, T.G. Papaioannou, K. Aberer, Synergies of different reputation systems: challenges and opportunities, August 25-27 (2009)

80. B. Walker, C.S. Holling, S.R. Carpenter, A. Kinzig, Resilience, adaptability and transformability in social-ecological systems. Ecology and Society, 9(2:5) http://www .ecologyandsociety.org/vol9/iss2/art5 (2004)

81. D. Walton, C. Reed, F. Macagno, Argumentation Schemes (Cambridge University Press, Cambridge, 2008)

82. C. Ware, Information Visualisation - Perception for Design (Academic Press, San Diego, CA, 2004)

83. K. Weick, Sensemaking in Organizations (Sage, Thousand Oaks, CA, 1995)

84. Y. Charalabidis, R. Kleinfeld, E. Loukis, S. Steglich, Systematically Exploiting Web 2.0 Social Media in Government for Extending Communication with Citizens (IGI Global, 2011) 